\title{
The influence of autotoxicity on the dynamics of vegetation spots
}

\author{
Annalisa Iuorio* \\ University of Vienna, Faculty of Mathematics, Oskar-Morgenstern-Platz, 1, 1090 Vienna, Austria. \\ Frits Veerman* \\ University of Leiden, Mathematical Institute, Niels Bohrweg 1, 2300 RA Leiden, The Netherlands.
}

\begin{abstract}
Plant autotoxicity has proved to play an essential role in the behaviour of local vegetation. We analyse a reactiondiffusion-ODE model describing the interactions between vegetation, water, and autotoxicity. The presence of autotoxicity is seen to induce movement and deformation of spot patterns in some parameter regimes, a phenomenon which does not occur in classical biomass-water models. We aim to analytically quantify this novel feature, by studying travelling wave solutions in one spatial dimension. We use geometric singular perturbation theory to prove the existence of symmetric, stationary and non-symmetric, travelling pulse solutions, by constructing appropriate homoclinic orbits in the associated 5-dimensional dynamical system. In the singularly perturbed context, we perform an extensive scaling analysis of the dynamical system, identifying multiple asymptotic scaling regimes where (travelling) pulses may or may not be constructed. We show that, while the analytically constructed stationary pulse corresponds to its numerical counterpart, there is a discrepancy between the numerically observed travelling pulse and its analytical counterpart. Our findings indicate how the inclusion of an additional ODE may significantly influence the properties of classical biomass-water models of Klausmeier/Gray-Scott type.
\end{abstract}

Keywords:

pattern formation; travelling pulses; vegetation patterns; geometric singular perturbation theory; autotoxicity; reaction-diffusion-ODE systems

\section{Introduction}

Self-organized vegetation patterning has been proved in recent years to play an important role in our understanding of climate change and catastrophic shifts [44], in particular in arid and semi-arid environments (e.g., [9, 10]). Several reasons have been hypothesised to explain the occurrence of such patterns, focusing on spatial interactions between vegetation and the abiotic environment (e.g., [11, 20, 23, 27, 45, 49, 50]). The main mechanisms that have been identified in vegetation self-organization rely on the transport of water and nutrients toward vegetated patches through a scale-dependent (short-range positive, long-range negative) feedback, allowing plants to act as ecosystems engineers [16, 32]. Water availability alone, however, fails to explain the emergence of vegetation patterns in environments where water availability is not limited (see [46] and references therein).

A mechanism that is by now well established in the description of vegetation dynamics is plant-soil negative feedback (see, e.g., [15, 34, 42]). This feedback, which was already well-known in agriculture since ancient time, underlies the practice of "crop rotation" after repeated monoculture to avoid "soil sickness" - a situation where the same plant species cannot be grown within the same region after a certain amount of time. The origin of this feedback has been linked to several biological phenomena, such as the presence of soilborne pathogens, the changing composition of soil microbial communities [31], and the accumulation of autotoxic compounds from decomposing plant litter [4, 39]. In particular, it has been proved that plant-soil negative feedback occurs because of inhibitory effects of extracellular DNA [40]. Extensive studies have revealed that this negative feedback plays an important role in several aspects of vegetation dynamics, such as species coexistence and biodiversity [38], as well as spatial organisation of plants by means of clonal rings [5, 6] and patterns [35].

In [35], in particular, the authors have found that the presence of autotoxicity induces - for some parameter ranges, and in agreement with experimental observations - the occurrence of dynamic patterns, with a non-symmetric

${ }^{*}$ Corresponding author

Email addresses: annalisa.iuorio@univie.ac.at (Annalisa Iuorio), f.w.j.veerman@math.leidenuniv.nl (Frits Veerman) 
bioRxiv preprint doi: https://doi.org/10.1101/2020.07.29.226522; this version posted June 24,2021 . The copyright holder for this preprint (which was not certified by peer review) is the author/funder. All rights reserved. No reuse allowed without permission.

distribution of biomass. The governing process is represented by the attempt of the biomass to "escape" areas with high levels of toxicity. The analytical foundation for such ecologically relevant results, however, is still lacking.

In this paper, we aim to take a first step in analytically justifying the numerical findings in [35] by using recently developed analytical techniques [14, 48]. In particular, we prove existence of both stationary and travelling pulses in one spatial dimension. To this aim, we use a convenient scaling, which allows us to transform the model introduced in [35] into an extension of the classical Klausmeier/Gray-Scott (KGS) model. For this classical KGS model, a large number of extensive analytical and numerical studies have been performed, investigating the existence, stability and dynamics of several types of patterns [12, 13, 28, 29, 41, 49, 50]; note that these references are but an incomplete selection of the vast literature on pattern formation in the KGS model.

We show that the extension of the KGS model with a third, spatially homogeneous, ordinary differential equation that models autotoxicity, can significantly influence the development and behaviour of pattern solutions, inducing the formation of travelling pulses which have been proven not to exist in the corresponding toxicity-free case [13].

From an analytical point of view, the inclusion of an additional model component significantly increases the complexity of the model, and hence introduces a potential obstacle for the successful analysis of pattern formation in this extended KGS model. One of the main results of this paper is that several asymptotic scaling regimes can be identified where the techniques developed in a two-component setting [14] nevertheless can be extended to analyse the existence of pulse patterns in this new three-component 'reaction-diffusion-ODE' model.

In the original KGS model, the existence and stability of pulse solutions sensitively depends on the asymptotic scaling of model components and parameters, see e.g. [2]. In order to analytically understand certain numerically observed behaviour, the 'proper' asymptotic scaling regime has to be identified. In the classical KGS model, this has already proven to be a subtle problem (see e.g. [48]); the inclusion of an additional model component exacerbates this subtility by increasing the number of scaling regimes that can be considered. Since choosing a particular asymptotic scaling is, to a certain extent, a prerequisite for the application of the analytical techniques presented in [14], the in-depth investigation of asymptotic scaling regimes relevant for investigating the existence of stationary and travelling pulse solutions, is a major part of this paper.

The paper is structured as follows: in Section 2, we present the original (dimensional) biomass-water-autotoxicity model [35], and the nondimensionalised, scaled model version we use in our subsequent analysis. Section 3 revolves around a collection of numerical results obtained by simulating both the original model in two spatial dimensions, and the rescaled, extended KGS model in a one-dimensional domain, showing the influence of autotoxicity. Section 4 is devoted to the existence for stationary and travelling pulses. Finally, a discussion of the presented results and an outline for future work in Section 5 conclude the paper.

\section{The model}

The original model introduced in [35] describes the dynamics of biomass $(B)$, water $(W)$, and autotoxicity $(T)$ on a two-dimensional spatial domain, all measured in $\mathrm{kg} \mathrm{m}^{-2}$, as follows: Growth of biomass $B$ is mediated by water availability, its intrinsic mortality, and the toxic compounds; availability of water $W$ is affected by precipitation, evaporation, and transpiration (plant water uptake); finally, autotoxicity $T$ grows due to the decomposition of dead plants and is removed from the soil by intrinsic degradation and precipitation. In mathematical terms, this corresponds to

$$
\begin{aligned}
& \frac{\partial W}{\partial t}=D_{W} \nabla_{\mathbf{x}}^{2} W+p-r B^{2} W-l W, \\
& \frac{\partial B}{\partial t}=D_{B} \nabla_{\mathbf{x}}^{2} B+c B^{2} W-(d+s T) B, \\
& \frac{\partial T}{\partial t}=q(d+s T) B-(k+w p) T,
\end{aligned}
$$

with time $t \geq 0$ measured in days (d) and space $\mathbf{x}=(x, y) \in \Omega \subset \mathbb{R}^{2}$ in meters $(\mathrm{m}) ; \nabla_{\mathbf{x}}^{2}$ is the usual planar Laplace operator. System 2.1] is an extension of the classical Klausmeier model [27] on flat terrain, hence the absence of an advection term in (2.1a) (see e.g. [26]). A detailed description of the model together with a biological interpretation of System [2.1] is given in [35]. For an overview of the model parameters as in [35] see Table 1. these values are either chosen in accordance with [27] and [6] or selected from within an order-of-magnitude feasibility range. 
bioRxiv preprint doi: https://doi.org/10.1101/2020.07.29.226522; this version posted June 24, 2021. The copyright holder for this preprint (which was not certified by peer review) is the author/funder. All rights reserved. No reuse allowed without permission.

\begin{tabular}{llll}
\hline Parameter & Description & Units & Value in [35] \\
\hline$c$ & Growth rate of $B$ due to water uptake & $\mathrm{m}^{4} \mathrm{~d}^{-1} \mathrm{~kg}^{-2}$ & $2 \cdot 10^{-3}$ \\
$d$ & Death rate of biomass $B$ & $\mathrm{~d}^{-1}$ & $1 \cdot 10^{-2}$ \\
$k$ & Decay rate of toxicity $T$ & $\mathrm{~d}^{-1}$ & $1 \cdot 10^{-2} \mathrm{or} 2 \cdot 10^{-1}$ \\
$l$ & Water loss due to evaporation or drainage & $\mathrm{d}^{-1}$ & $1 \cdot 10^{-2}$ \\
$p$ & Precipitation rate & $\mathrm{kg} \mathrm{d}^{-1} \mathrm{~m}^{-2}$ & {$[0,2]$} \\
$q$ & Proportion of toxins in dead biomass & - & $5 \cdot 10^{-2}$ \\
$r$ & Rate of water uptake & $\mathrm{m}^{4} \mathrm{~d}^{-1} \mathrm{~kg}^{-2}$ & $3.5 \cdot 10^{-1}$ \\
$s$ & Sensitivity of plants to toxicity $T$ & $\mathrm{~m}^{2} \mathrm{~d}^{-1} \mathrm{~kg}^{-1}$ & $0 \mathrm{or} 2 \cdot 10^{-1}$ \\
$w$ & Washing out of toxins by precipitation & $\mathrm{m}^{2} \mathrm{~kg}^{-1}(*)$ & $1 \cdot 10^{-3}$ \\
$D_{B}$ & Diffusion coefficient of biomass $B$ & $\mathrm{~m}^{2} \mathrm{~d}^{-1}$ & $1 \cdot 10^{-2}$ \\
$D_{W}$ & Diffusion coefficient of water $W$ & $\mathrm{~m}^{2} \mathrm{~d}^{-1}$ & $8 \cdot 10^{-1}$ \\
\hline
\end{tabular}

Table 1: Model parameters for model [2.1] as presented in [35]. Note $(*)$ : the dimension of $w$ was stated incorrectly in [35]. ables

We rescale and nondimensionalise [2.1] as follows. First, we define the nondimensional space and time vari-

$$
\tilde{\mathbf{x}}:=\frac{c p}{l \sqrt{D_{W} r}} \mathbf{x}, \quad \tilde{t}:=\frac{c^{2} p^{2}}{l^{2} r} t .
$$

Next, we define the nondimensional model components

$$
U(\tilde{\mathbf{x}}, \tilde{t}):=\frac{l}{p} W(\mathbf{x}, t), \quad V(\tilde{\mathbf{x}}, \tilde{t}):=\frac{l r}{c p} B(\mathbf{x}, t), \quad S(\tilde{\mathbf{x}}, \tilde{t}):=\frac{(k+p w) l^{3} r^{2}}{c^{3} p^{3} q} T(\mathbf{x}, t) .
$$

The model in the new components $(U, V, S)$ reads

$$
\begin{aligned}
\frac{\partial U}{\partial \tilde{t}} & =\nabla_{\tilde{\mathbf{x}}}^{2} U+\mathcal{A}(1-U)-U V^{2}, \\
\frac{\partial V}{\partial \tilde{t}} & =\varepsilon^{2} \nabla_{\tilde{\mathbf{x}}}^{2} V+U V^{2}-\mathcal{B} V-\mathcal{H} V S, \\
\mathcal{D} \frac{\partial S}{\partial \tilde{t}} & =-S+\mathcal{B} V+\mathcal{H} V S,
\end{aligned}
$$

defined on a two-dimensional spatial domain $\tilde{\Omega} \subset \mathbb{R}^{2}$ for $\tilde{t} \geq 0$. The (nondimensional) parameters in $(2.4)$ are defined as

$$
\mathcal{A}:=\frac{l^{3} r}{c^{2} p^{2}}, \quad \mathcal{B}:=\frac{d l^{2} r}{c^{2} p^{2}}=\frac{d}{l} \mathcal{A}, \quad \mathcal{D}:=\frac{c^{2} p^{2}}{(k+p w) l^{2} r}=\frac{d}{k+p w} \frac{1}{\mathcal{B}}, \quad \mathcal{H}:=\frac{c p q s}{(k+p w) l r}=\frac{q s l}{c p} \mathcal{D},
$$

while the (square root of the) diffusivity ratio gives rise to the natural small parameter

$$
\varepsilon:=\sqrt{\frac{D_{B}}{D_{W}}} .
$$

Note that decoupling the autotoxicity equation by setting $\mathcal{H}=0$ is equivalent to taking the toxicity sensitivity $s=0$.

The original KGS model can be recovered by setting $\mathcal{H}=0$ in $(2.4$, thereby decoupling the toxicity variable $S$ from the water-biomass variable pair $(U, V)$. For the decoupled system, there is extensive literature on the existence and dynamics of several types of patterns, both in one and two spatial dimensions (e.g. [2, 7, 12, 13, 28, 29, 41, 48, 49, 50, 54]). In particular, the existence and dynamics of pulse solutions in the original KGS model has been studied in great detail, using a variety of techniques [12, 13, 28, 29, 41, 49, 50]. In this paper, we adopt the analytical approach as outlined in [14]. Moreover, model (2.4) has been rescaled to enable parameter identification with the original KGS model as analysed in [13]. We want to emphasise that this does not imply our model formulation is in any way preferable over alternative formulations, such as those used in [28, 29] or [49, 50]; our choice merely reflects the similarity to the method employed in [13, 48], to enable easy comparison with previous results.

\section{Numerics}

In this section we illustrate the results obtained by performing numerical simulations of equations (2.1) and (2.4) on a two-dimensional and one-dimensional spatial domain, respectively. The main aim of this section is to 
bioRxiv preprint doi: https://doi.org/10.1101/2020.07.29.226522; this version posted June 24, 2021. The copyright holder for this preprint (which was not certified by peer review) is the author/funder. All rights reserved. No reuse allowed without permission.

highlight the differences caused by the presence of toxicity when compared to 'classical' biomass-water models without negative plant-soil feedback. When such negative plant-soil feedback is particularly strong (i.e. for low precipitation rate, high sensitivity to toxicity, and low toxicity decay rate), two effects arise, which are not detected in biomass-water models without toxicity: the emergence of spatio-temporal (dynamic) patterns and an asymmetric biomass distribution within the patterns.

\subsection{Simulations on a two-dimensional domain}

We consider the dimensional biomass-water-toxicity model 2.1 on a two-dimensional domain $\Omega=\left\{0 \leq x \leq L_{x}\right.$, $\left.0 \leq y \leq L_{y}\right\}$ with boundary and initial conditions

$$
\partial_{n} W=0, \quad \partial_{n} B=0, \quad \mathbf{x} \in \partial \Omega, \quad t \geq 0
$$

and

$$
W(\mathbf{x}, 0)=W_{0}(\mathbf{x}), \quad B(\mathbf{x}, 0)=B_{0}(\mathbf{x}), \quad T(\mathbf{x}, 0)=0, \quad \mathbf{x} \in \Omega
$$

respectively. Here, $\partial \Omega$ is the boundary of $\Omega, \partial_{n}$ is the normal derivative on $\partial \Omega, B_{0}$ and $W_{0}$ are initial spatial distributions of biomass and water, respectively. Our simulations are based on a numerical scheme which uses finite differences in space, and forward Euler in time. The two-dimensional domain $\Omega$ is discretised via a square lattice of $100 \times 100$ elements, with $\Delta x=\Delta y=1$ and $L_{x}=L_{y}=99$ meters. The initial datum for the biomass $B_{0}(\mathbf{x})$ satisfies $B_{0}=0.2$ in $N_{0}=5000$ randomly selected elements (total initial biomass $B_{0}^{\text {tot }}=1000$ ) and $B_{0}=0$ in the remaining nodes, while $W_{0}(\mathbf{x})=40$ uniformly. The simulation time $t_{\max }=1000$ days consists of $10^{5}$ time-steps $\Delta t$, with $\Delta t=0.01$ days.

Since our goal is to investigate the difference between the case without toxicity $(s=0)$ and the one with strong influence of toxicity $(s \neq 0$, small $k$ ), we fix all other parameter values in (2.1) as follows

$$
c=0.002, d=0.01, l=0.01, p=0.65, q=0.05, r=0.35, w=0.001, D_{B}=0.01, D_{W}=0.8,
$$

and consider two particularly representative cases for $s$ and $k$ (see Figure 11). In the case without toxicity ( $s=0$, Figure 1(a)), numerical simulations show the emergence of a stable stationary spatial pattern in the form of spots, consistent with existing literature results (see e.g. [26]). On the other hand, for $s=0.3$ and $k=0.01$, strong negative feedback due to plant toxicity induces the formation of dynamic patterns that continuously evolve in time with a propagation speed of about 3 meter/year. Moreover, the biomass distribution within the pattern is not symmetric, yielding crescent moon shapes (see Figure 1 (b)). These crescent moon shapes are observed to travel with constant velocity in the direction of highest local biomass concentration. Moreover, the velocity distribution is seen to be isotropic. Such non-symmetric patterns are usually linked to the presence of an advection term in the water equation, which models water transport due to slope (see e.g. [52, Figure 11]). Negative feedback due to autotoxicity is hence able to reproduce such non-symmetric shapes on flat terrain, in agreement with experimental observations (see [35]).

\subsection{Simulations on a one-dimensional domain}

We consider equations the nondimensionalised, rescaled model 2.4 on a one-dimensional domain $\tilde{\Omega}=$ $\{0 \leq \tilde{x} \leq L\}$ with boundary and initial conditions

$$
\partial_{n} U=0, \quad \partial_{n} V=0, \quad \tilde{x} \in \partial \tilde{\Omega}, \quad \tilde{t} \geq 0
$$

and

$$
U(x, 0)=U_{0}(\tilde{x}), \quad V(x, 0)=V_{0}(\tilde{x}), \quad S(\tilde{x}, 0)=0, \quad \tilde{x} \in \tilde{\Omega} .
$$

Our numerical simulations are again based on a numerical scheme which uses finite differences in space, and forward Euler in time. We consider $\tilde{\Omega}=[0,32]$ and discretize the domain by fixing $m=801$ nodes at equal distance $\Delta \tilde{x}=0.04$. The simulation time $\tilde{t}_{\max }=2000$ consists of $10^{7}$ time steps $\Delta \tilde{t}$, with $\Delta \tilde{t}=2 \cdot 10^{-4}$.

For $\mathcal{H}=0$, system 2.4 reduces to the classical KGS model. In one spatial dimension, the KGS model has been extensively studied, revealing the formation of self-replicating patterns (see e.g. [13, 28, 43]). In particular, in [13], the non-existence of travelling solitary pulses and the occurrence of pulse splitting leading to a stationary periodic pulse pattern have been shown, both analytically and numerically. In this section, our aim is to compare the numerical results presented in [13] for the toxicity-free scenario, with the scenario where (2.4b) and (2.4c) are coupled (i.e., $\mathcal{H}=0$ versus $\mathcal{H} \neq 0$ ). To this end, we fix all parameters except for $\mathcal{H}$ as follows:

$$
\mathcal{A}=\mathcal{B}=0.2, \quad \mathcal{D}=4.5, \quad \varepsilon=0.1 \text {. }
$$




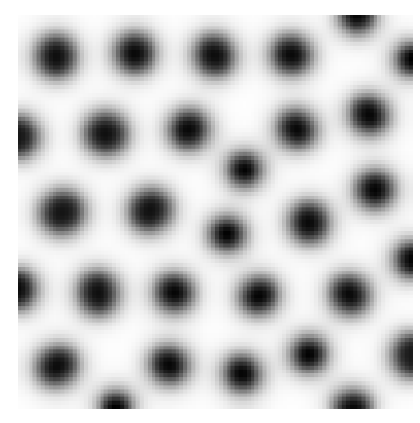

(a)

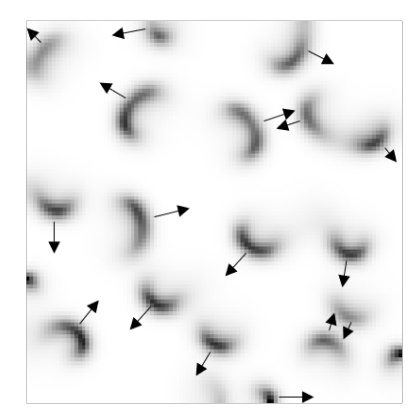

(d)

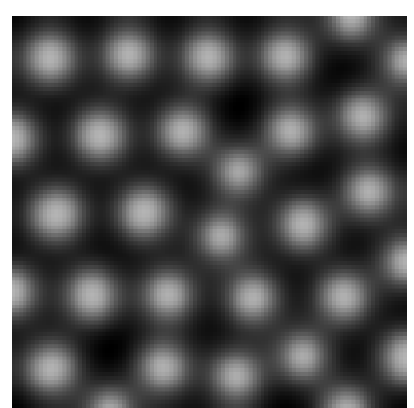

(b)

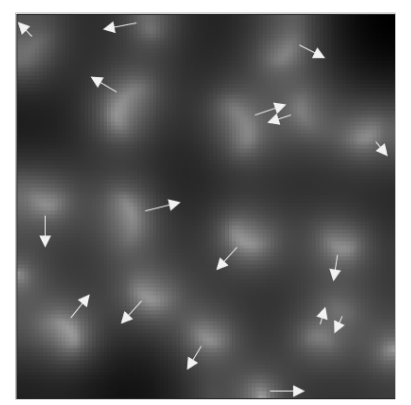

(e)

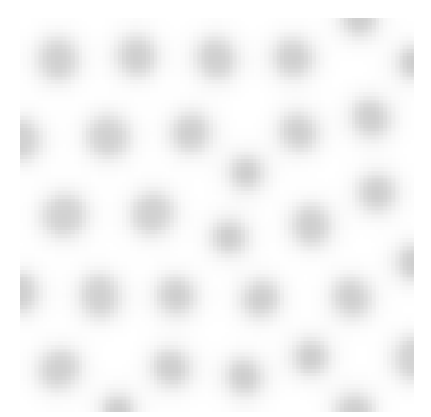

(c)

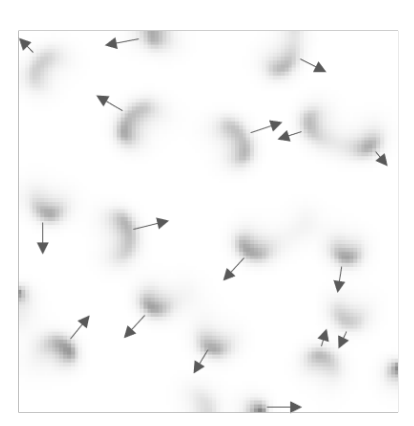

(f)

Figure 1: Biomass patterns obtained simulating equations 2.1. with parameter values [3.2, initial conditions and boundary conditions as in 3.1) at $t=t_{\max }$. Darker shades of gray correspond to higher density of $B\left(\mathbf{x}, t_{\max }\right), W\left(\mathbf{x}, t_{\max }\right)$, and $T\left(\mathbf{x}, t_{\max }\right)$. The first row corresponds to simulations with $s=0$, any $k$ for (a) $B$, (b) $W$, and (c) $T$. In this case, the influence of toxicity is absent (as equations 2.1b) and 2.1c are decoupled), and we recover (away from the boundaries) the symmetric stationary spots present in 'classical' biomass-water models (see [26 27]). The second row corresponds to simulations with $s=0.3, k=0.01$ for (d) $B$, (e) $W$, and (f) $T$. Toxicity induces both a non-symmetric distribution of biomass within the spots, and movement of the spots (indicated by the arrows) with constant velocity - that is, the formation of a dynamic spatio-temporal pattern. Movies illustrating the above dynamics are available in the supplementary material of [35].

These values are commensurate with the parameter choices in the two-dimensional simulations $(3.2)$, with in addition $k=0.01$, and $s=0$ (no toxicity sensitivity) or $s=0.3$ (high toxicity sensitivity). As initial data, we take $U_{0}(\tilde{x})=0.62$ uniformly, which is commensurate with the choice $W_{0}(\mathbf{x})=40$ made in subsection 3.1 . For $V$, we consider the following two initial profiles:

- a Gaussian peak in the centre of the domain

$$
V_{0}(\tilde{x})=\frac{2}{10 \sqrt{2 \pi}} e^{-\frac{1}{2}(5(\tilde{x}-16) / 2)^{2}}
$$

- a "half-bare-half-vegetated" domain

$$
V_{0}(\tilde{x})= \begin{cases}0.6 & 16<\tilde{x}<\frac{797}{25} \\ 0 & \text { otherwise }\end{cases}
$$

(We note that the condition $V_{0}=0$ at $\tilde{x}=32$ is motivated by our intent to analyse the effect of different boundary conditions while keeping the same initial configuration for $V$.) When $\mathcal{H}=0$ (see Figure 2), we observe the formation of a stationary, periodic, spotted pattern as a consequence of pulse splitting, independent of the choice of the initial $V$-profile. This observation is in agreement with the results presented in [13, 26] and with the twodimensional numerical simulations presented in subsection 3.1. When we now 'turn on' the influence of toxicity by choosing $\mathcal{H}=0.5$, the numerical simulations show the emergence of travelling pulses. In particular, when the initial profile $V_{0}(\tilde{x})$ is chosen as in (3.5), the initial pulse symmetrically splits into two pulses which periodically move away from each other and toward each other within the domain (see Figure 3 (a)). On the other hand, if we take the "half-vegetated-half-bare" initial profile (3.6), we observe that a single pulse forms, which periodically travels back and forth within the domain (see Figure 3 (b)). In both cases, numerical results reveal that the travelling pulse is asymmetric (see Figure 4) and that the speed of such pulses is approximately 0.0384, which could be interpreted $O\left(\varepsilon^{2}\right)$ for this choice of $\varepsilon$. This value is of the same order of magnitude with respect to the numerical propagation speed computed for the $2 \mathrm{D}$ simulations.

A snapshot of the simulated travelling pulse is shown in Figure 5 
bioRxiv preprint doi: https://doi.org/10.1101/2020.07.29.226522; this version posted June 24, 2021. The copyright holder for this preprint (which was not certified by peer review) is the author/funder. All rights reserved. No reuse allowed without permission.

Remark 3.1 (Boundary conditions). The results presented in this section appear to be independent of boundary conditions, as the travelling pulse structure persists when considering different (larger) domains which mimick an infinite spatial interval. We are mainly interested in the dynamics occurring away from the boundaries: therefore, even though the interaction with the boundary might lead to some transient effects, (see e.g. Fig. 3), their relevance in our analysis can be neglected.

Remark 3.2 (Simulations time). Numerical evidence strongly suggests that the findings presented in Section 3.1 and 3.2 are not affected by the length of the simulations times, i.e. they persist when considering longer intervals mimicking long-time behaviour. Therefore, we focus our attention on finite times, with $t_{\max }=1000$ days for $2 \mathrm{D}$ simulations and $\tilde{t}_{\max }=2000$ in the case of $1 \mathrm{D}$ simulations.

Remarks 3.1 and 3.2 suggest that the travelling pulse observed in the simulations is stable in the PDE sense.

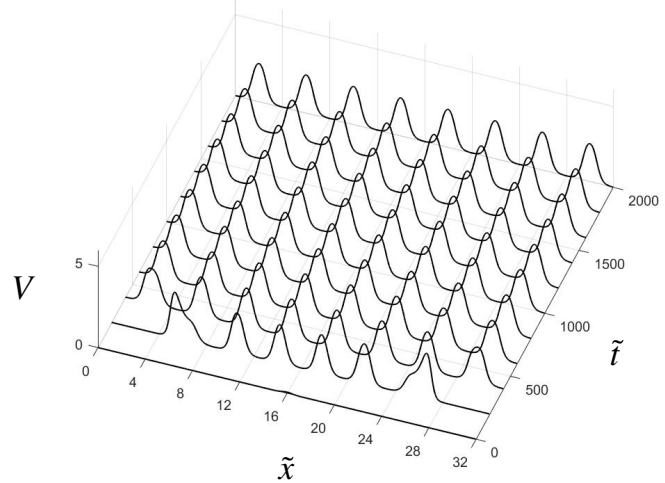

(a)

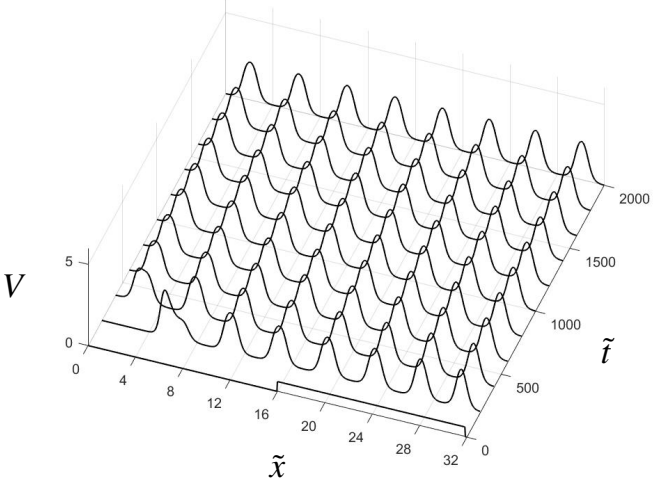

(b)

Figure 2: Evolution of $V$ in space $\tilde{x}$ and time $\tilde{t}$ obtained by numerically simulating equations 2.4 with parameters as in 3.4 and $\mathcal{H}=0$. (a) Simulations obtained for an initial datum $V_{0}(\tilde{x})$ as in 3.5. (b) Here, $V_{0}(\tilde{x})$ is fixed as in 3.6. In both cases, decoupling the dynamics of $V$ from those of $S$ leads to a symmetric, stable, regular pulse pattern through a process of pulse splitting.

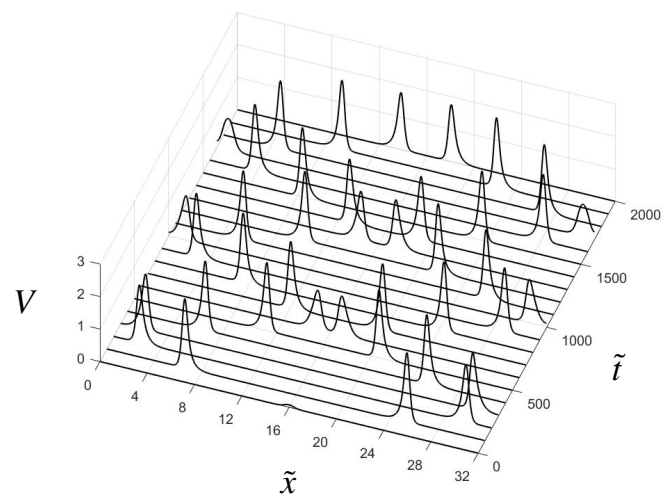

(a)

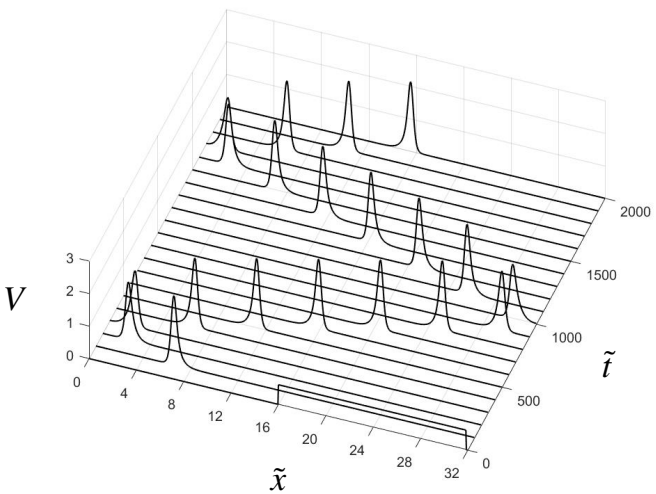

(b)

Figure 3: Evolution of $V$ in space $\tilde{x}$ and time $\tilde{t}$ obtained by numerically simulating equations 2.4 with parameters as in 3.4 and $\mathcal{H}=0.5$. (a) Simulations obtained for an initial datum $V_{0}(\tilde{x})$ as in 3.5 . The strong influence of $S$ on the dynamics of $V$ induces the formation of a symmetric pair of pulses, which travel within the domain, moving away from and towards each other periodically. (b) Here, $V_{0}(\tilde{x})$ is chosen as in 3.6. In this case, a single pulse forms, which travels back and forth along the spatial domain. Note that, in both cases, $V$ is not symmetrically distributed within the pulse. 
(a)

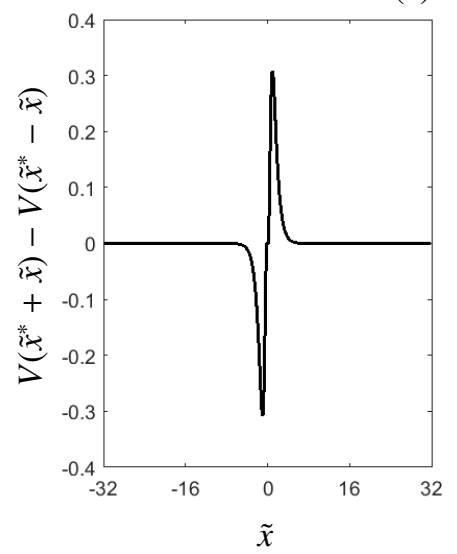

(b)

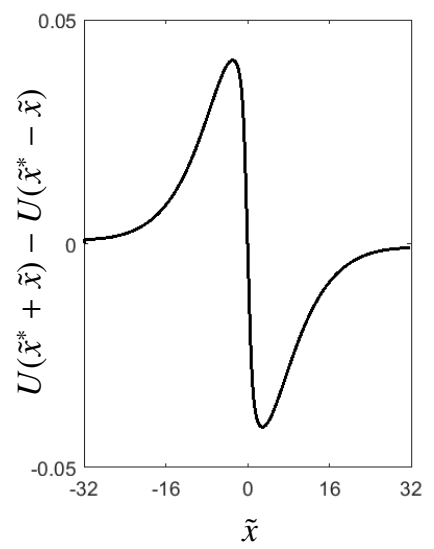

(c)

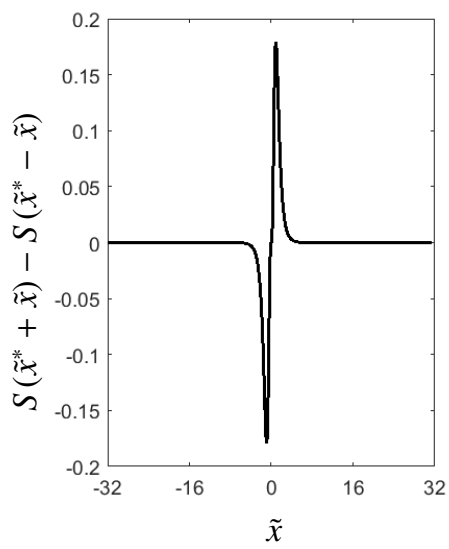

Figure 4: Measurement of asymmetry of the travelling pulse obtained by simulating system 2.4) until time $\tilde{t}=600$, starting from $V_{0}(\tilde{x})$ as in 3.6. The symbol $\tilde{x}^{*}$ indicates the $x$-value where $V$ reaches its maximum value (peak of the pulse). The panels show the asymmetry function $Q\left(\tilde{x}^{*}+\tilde{x}\right)-Q\left(\tilde{x}^{*}-\tilde{x}\right)$, where (a) $Q=V$, (b) $Q=U$, and (c) $Q=S$.

(a)

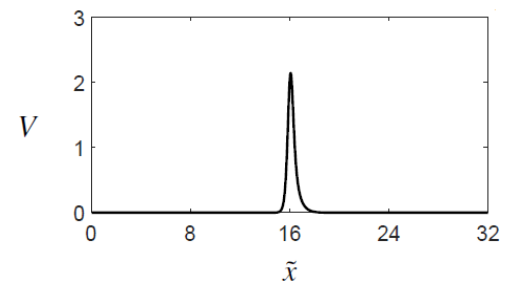

(b)

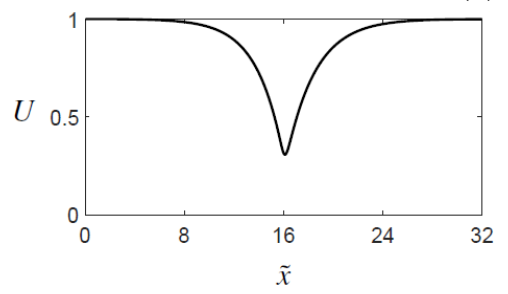

(c)

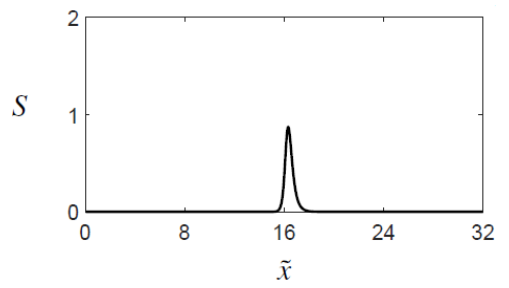

Figure 5: Snapshot of the a travelling pulse at time $\tilde{t}=600$ obtained by simulating system 2.4 starting from $V_{0}(\tilde{x})$ as in 3.6 with parameters as in 3.4. (a) $V$-component, (b) $U$-component, (c) $S$-component.

A preliminary, order-of-magnitude investigation into the role of the parameters $\mathcal{A}, \mathcal{B}$, and $\mathcal{D}$ is presented in Table 2. An important observation is that the existence of pulse solutions not only clearly depends on the magnitude of $\mathcal{A}$ and $\mathcal{B}$, but also on the fact that $\mathcal{A}$ and $\mathcal{B}$ have to be chosen (approximately) equal. Secondly, by varying $\mathcal{D}$, we are able to find stationary pulse solutions. In addition, for relatively low values of $\mathcal{A}, \mathcal{B}$ and $\mathcal{D}$, simulations show strongly asymmetric, travelling pulse solutions -or travelling fronts with a decaying back- as a transient phase towards bare soil, see Figure 6. To our knowledge, transients of this shape have not been observed in the context of the classical KGS model.

\begin{tabular}{|c|l|l|l|}
\hline & $\mathcal{D}=0.04$ & $\mathcal{D}=0.4$ & $\mathcal{D}=4.5$ \\
\hline $\mathcal{A}, \mathcal{B}=0.02$ & Bare soil; see Figure 6 & Bare soil & Bare soil \\
\hline $\mathcal{A}, \mathcal{B}=0.2$ & $\begin{array}{l}(*) \text { Travelling pulse(s), ap- } \\
\text { proximate speed } 0.0042\end{array}$ & Stationary pulse(s) & $\begin{array}{l}\text { Travelling pulse(s), approxim- } \\
\text { ate speed 0.0384; see Figure } 3\end{array}$ \\
\hline $\mathcal{A}, \mathcal{B}=2$ & Bare soil & Bare soil & Bare soil \\
\hline $\mathcal{A} \neq \mathcal{B}$ & Bare soil & Bare soil & Bare soil \\
\hline
\end{tabular}

Table 2: An overview of numerical simulation results of system 2.4 for several choices of parameters $\mathcal{A}, \mathcal{B}$ and $\mathcal{D}$. Throughout, $\mathcal{H}=0.5$ and $\varepsilon=0.1$. (*) In the case $\mathcal{A}=\mathcal{B}=0.2, \mathcal{D}=0.04$, the pulses seem to be slowing down over the simulated time interval and its travelling speed tends to zero when extending the simulation to $t=100000$, leading to a stationary pulse. 


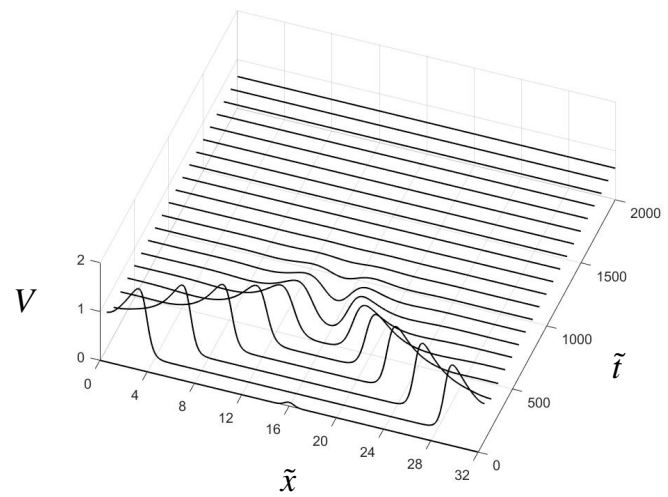

(a)

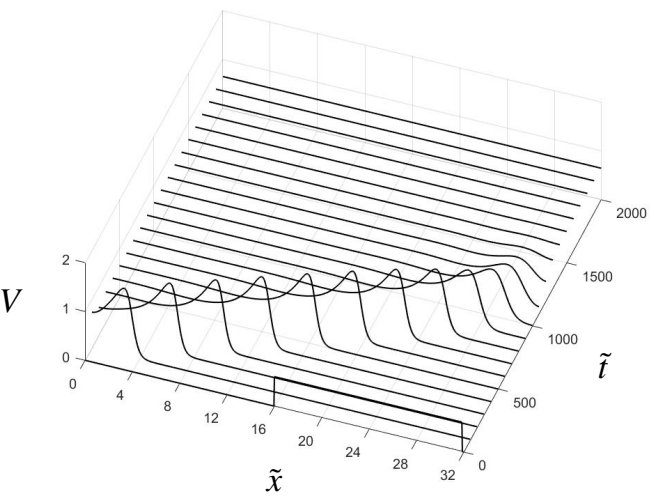

(b)

Figure 6: Evolution of $V$ in space $\tilde{x}$ and time $\tilde{t}$ obtained by numerically simulating equations $(2.4)$ with $\mathcal{A}=\mathcal{B}=0.02, \mathcal{D}=0.4, \mathcal{H}=0.5$, and $\varepsilon=0.1$. (a) Initial datum $V_{0}(\tilde{x})$ as in 3.5 . Here, two travelling fronts form, meet at the centre of the domain, and then merge and decay to a bare soil state. (b) $V_{0}(\tilde{x})$ as in (3.6). In this case, a single travelling front emerges, and eventually decays to the bare soil state as well.

\section{Existence of pulse solutions}

In this section, we investigate the existence of stationary and travelling pulse solutions to system (2.4) on an unbounded, one-dimensional spatial domain $\tilde{\Omega}=\mathbb{R}$. We confine our study to pulse solutions that are biasymptotic to the trivial homogeneous stable steady (bare soil) state $(U, V, W)=(1,0,0)$. System (2.4) in one spatial dimension is restated here for completeness:

$$
\begin{aligned}
\frac{\partial U}{\partial \tilde{t}} & =U_{\tilde{x} \tilde{x}}+\mathcal{A}(1-U)-U V^{2}, \\
\frac{\partial V}{\partial \tilde{t}} & =\varepsilon^{2} V_{\tilde{x} \tilde{x}}+U V^{2}-\mathcal{B} V-\mathcal{H} V S, \\
\mathcal{D} \frac{\partial S}{\partial \tilde{t}} & =-S+\mathcal{B} V+\mathcal{H} V S .
\end{aligned}
$$

Remark 4.1 (Homogeneous steady states). Apart from the trivial homogeneous steady state $(U, V, S)=(1,0,0)$, system (4.1) admits two nontrivial homogeneous steady states, given by

$$
\left(U_{ \pm}, V_{ \pm}, S_{ \pm}\right)=\left(1-\frac{S_{ \pm}}{A}, \frac{1 \pm \sqrt{1-4 B\left(\frac{B}{A}+H\right)}}{2\left(\frac{B}{A}+H\right)}, \frac{B V_{ \pm}}{1-H V_{ \pm}}\right),
$$

which exist for $0<B<\frac{1}{2 H}$ and $\frac{1}{1-2 B H}<\frac{A}{2 B^{2}}$. The limit $H \rightarrow 0$ yields the pair of nontrivial homogeneous steady states in the original Klausmeier model, with existence condition $\frac{A}{2 B^{2}}>1$. However, one can choose $A$ and $B$ such that the nontrivial solution pair (4.2) does not exist for $H$ below (alternatively, above) a certain threshold value. Hence, the family (4.2) is not for every $A, B$ and $H$ a continuous extension of the pair of nontrivial steady states in the original Klausmeier model.

We introduce a co-moving frame coordinate

$$
\tilde{z}=\tilde{x}-C \tilde{t},
$$

in which system (4.1) takes the form

$$
\begin{aligned}
& 0=U_{\tilde{z} \tilde{z}}+\mathcal{A}(1-U)-U V^{2}+C U_{\tilde{z}}, \\
& 0=\varepsilon^{2} V_{\tilde{z} \tilde{z}}+U V^{2}-\mathcal{B} V-\mathcal{H} V S+C V_{\tilde{z}}, \\
& 0=C \mathcal{D} S_{\tilde{z}}-S+\mathcal{B} V+\mathcal{H} V S .
\end{aligned}
$$

We look for pulse solutions, i.e. solutions to (4.4) that are bi-asymptotic to the trivial background state $(U, V, S)=$ $(1,0,0)$, that is, solutions $(U(z), V(z), S(z))$ for which $\lim _{z \rightarrow \pm \infty}(U(z), V(z), S(z))=(1,0,0)$. To that end, we make the crucial assumption that $\varepsilon$, which measures the biomass diffusion rate with respect to the water diffusion rate, is an asymptotically small parameter, i.e. $0<\varepsilon \ll 1$. This is consistent with the experimental data collected in arid 
environments (see, e.g., [45] and references therein). Next, in accordance with previous work [12, 13, 48, 54], we allow all model variables and parameters to scale with (a power of) this small parameter $\varepsilon$. While the introduction of these scalings can seem unnecessary complicated and confusion due to the large number of new parameters that are introduced as scaling exponents, previous analysis [12, 13, 48, 54] has shown that several solution types -and pulse patterns in particular- exist only in regions of parameter space that scale with $\varepsilon$ in a particular way; moreover, the resulting pattern amplitude may also scale with $\varepsilon$. A preparatory asymptotic scaling analysis of system (4.4), along the lines of [13, 48], can be found in AppendixA. The resulting rescaling can be summarised as follows:

$$
\begin{array}{r}
\mathcal{A}=\varepsilon^{2+3 \beta-2 \kappa} a, \mathcal{B}=\varepsilon^{\beta} b, C=\varepsilon^{\gamma} c, \mathcal{D}=\varepsilon^{\delta} d, \mathcal{H}=\varepsilon^{\kappa-\beta} h, \\
U=\varepsilon^{\kappa} u, V=\varepsilon^{\beta-\kappa} v, S=\varepsilon^{2 \beta-\kappa} s, \tilde{z}=\varepsilon^{1-\frac{1}{2} \beta} z,
\end{array}
$$

with the additional assumptions that

$$
2+\beta-2 \kappa>0, \kappa>0 \text { and } \gamma>1+\frac{1}{2} \beta .
$$

Note that the positivity of the original model parameters $\mathcal{A}, \mathcal{B}, \mathcal{D}, \mathcal{H}$ implies that their rescaled counterparts $a, b, d, h$ are positive and $O(1)$ in $\varepsilon$; however, the associated scaling exponents may be negative. This also applies to the model variables $U, V, S$ and their rescaled counterparts $u, v, s$. The speed of the moving frame, $C$, can take any sign.

Application of rescaling (4.5) to (4.4) yields the following 5-dimensional dynamical system:

$$
\begin{aligned}
u_{z} & =\varepsilon^{2+\beta-2 \kappa} p, \\
p_{z} & =u v^{2}-\varepsilon^{2+\beta} a\left(\varepsilon^{-\kappa}-u\right)-\varepsilon^{1+\gamma-\frac{1}{2} \beta} c p, \\
v_{z} & =q, \\
q_{z} & =b v-u v^{2}+h v s-\varepsilon^{\gamma-\frac{1}{2} \beta-1} c q, \\
c d \varepsilon^{\gamma+\delta+\frac{1}{2} \beta-1} s_{z} & =s-b v-h v s .
\end{aligned}
$$

Pulse solutions to (4.4) can now be identified with orbits in system (4.7) that are homoclinic to the (rescaled) trivial background state $\left(\varepsilon^{-\kappa}, 0,0,0,0\right)$. In the following, we will use techniques from geometric singular perturbation theory [12, 13, 14, 48] to constructively establish the existence of such homoclinic orbits in system (4.7).

\subsection{Stationary pulses}

For stationary pulses, we have $C=0$ in 4.3, hence $c=0$ in 4.7). We obtain the differential-algebraic system

$$
\begin{aligned}
u_{z} & =\varepsilon_{1} p, \\
p_{z} & =u v^{2}-\varepsilon_{1} \varepsilon_{2} a\left(1-\varepsilon_{2} u\right), \\
v_{z} & =q, \\
q_{z} & =b v-u v^{2}+h v s, \\
0 & =s-b v-h v s,
\end{aligned}
$$

where we have defined the two small parameters

$$
\varepsilon_{1}:=\varepsilon^{2+\beta-2 \kappa} \text { and } \quad \varepsilon_{2}:=\varepsilon^{\kappa} .
$$

Note that $\varepsilon_{1,2}$ are indeed asymptotically small by the scaling conditions $\left(4.6\right.$. However, the case $\varepsilon_{2}=1$ is also covered by the analysis presented in this paper, but has been omitted for presentation purposes. For more information, we refer to AppendixA in paricular the remark below A.10].

First, we observe that the algebraic equation $4.8 \mathrm{e}$ can be solved for $s$, yielding

$$
s=\frac{b v}{1-h v} .
$$

From the biological interpretation of the model, it follows that all model components are assumed to be nonnegative, which implies that

$$
h v<1 \text {. }
$$

In other words, the dynamics of (4.8) take place on the 4-dimensional invariant manifold $\mathcal{M}_{0}$ embedded in 5dimensional $(u, p, v, q, s)$-phase space, that is given by

$$
\mathcal{M}_{0}:=\left\{(u, p, v, q, s) \in \mathbb{R}^{5} \mid s-b v-h v s=0, h v<1\right\} ;
$$


see also Figure 9 (a). At this point, the introduction of $\mathcal{M}_{0}$ can seem somewhat superfluous; however, this manifold will also play a role in the upcoming analysis of travelling pulses (see Section 4.2), and its introduction can illuminate similarities between the analysis of the current section and that of section 4.2

On $\mathcal{M}_{0}$, the dynamics are given by the 4-dimensional dynamical system

$$
\begin{aligned}
& u_{z}=\varepsilon_{1} p, \\
& p_{z}=u v^{2}-\varepsilon_{1} \varepsilon_{2} a\left(1-\varepsilon_{2} u\right), \\
& v_{z}=q, \\
& q_{z}=b v-u v^{2}+\frac{b h v^{2}}{1-h v} .
\end{aligned}
$$

Note that, for $h=0$, this is exactly the system studied in [12, 13]. For future reference, we introduce the 2dimensional hyperplane

$$
C_{0}:=\left\{(u, p, v, q, s) \in \mathbb{R}^{5} \mid v=0, q=0, s=0\right\} .
$$

We note that $C_{0}$ is invariant under the flow of (4.8); moreover, $C_{0} \subset \mathcal{M}_{0}$ (4.12).

Remark 4.2 (Fast and slow variables). In system (4.13), the p-variable cannot a priori be identified as 'slow' due to the presence of the $u v^{2}$-term in (4.13b). However, this does not preclude the application of geometric singular perturbation theory; specifically, from the analysis presented below and in previous work [12, 13, 14, 47, 48], it follows that the $p$-component expresses both slow and fast behaviour.

\subsubsection{Fast dynamics}

Following the approach of geometric singular perturbation theory, we study the fast reduced limit of (4.13) by letting $\varepsilon_{1} \rightarrow 0$, and obtain the planar, Hamiltonian, fast reduced system

$$
\begin{aligned}
u & =u_{0}=\text { constant } \\
v_{z} & =q, \\
q_{z} & =b v-u v^{2}+\frac{b h v^{2}}{1-h v} .
\end{aligned}
$$

The dynamics of $p$ can be obtained by direct integration of $p_{z}=u_{0} v^{2}$. Note that $C_{0}(4.14)$ consists of hyperbolic (trivial) equilibria of the fast reduced system 4.15]; hence, $C_{0}$ is a normally hyperbolic invariant manifold [30]. We introduce

$$
y:=\sqrt{b} z, \quad w(y):=h v(z)
$$

to obtain

$$
w_{y y}-\frac{w}{1-w}+\frac{1}{\delta} w^{2}=0, \quad \delta=\frac{b h}{u_{0}},
$$

with Hamiltonian

$$
H_{f}\left(w, w_{y}\right)=\frac{1}{2} w_{y}^{2}+w+\log (1-w)+\frac{1}{3 \delta} w^{3} .
$$

Analogously to [12, 13, 14, 48], we look for a homoclinic orbit to the origin in system (4.17); note that this orbit lies on the level set $H_{f}=0$. Straightforward phase plane analysis reveals that such a homoclinic orbit exists as long as $0<\delta<\delta_{\max }$, and the maximally attained $w$-value of the associated 'spike' solution is given by the unique positive solution to

$$
H_{f}(w, 0)=w+\log (1-w)+\frac{1}{3 \delta} w^{3}=0 .
$$

The value for $\delta_{\max }$ can now be determined by considering the situation when 4.19) is degenerate, which is when $\delta=w(1-w)$. This degeneracy occurs when the graph of $H_{f}(w, 0)$ is tangent to the $w$-axis for the unique positive solution to 4.19, causing the homoclinic orbit to deteriorate in a pair of heteroclinic orbits, as shown in Figure 7 (b). Hence, we find that

$$
\delta_{\max }=w_{\max }\left(1-w_{\max }\right),
$$

where $w_{\max }$ is the unique positive solution to

$$
w+\frac{1}{3} \frac{w^{2}}{1-w}+\log (1-w)=0
$$

the approximate numerical values are

$$
\delta_{\max }=0.228973, \quad w_{\max }=0.645007 .
$$




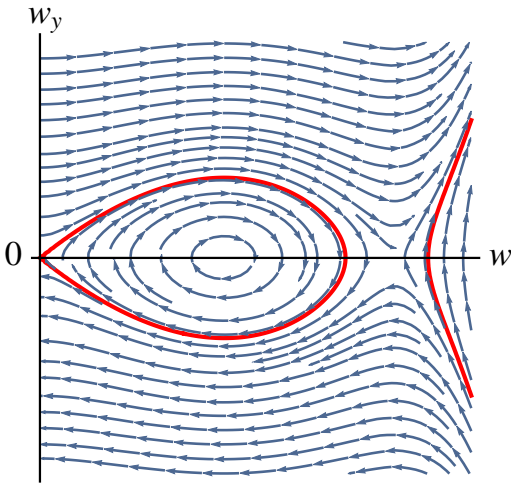

(a)

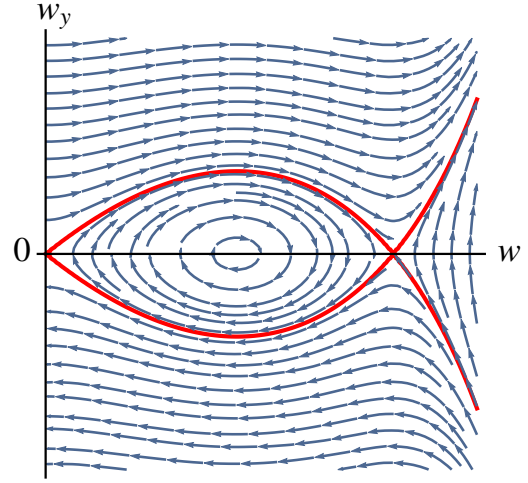

(b)

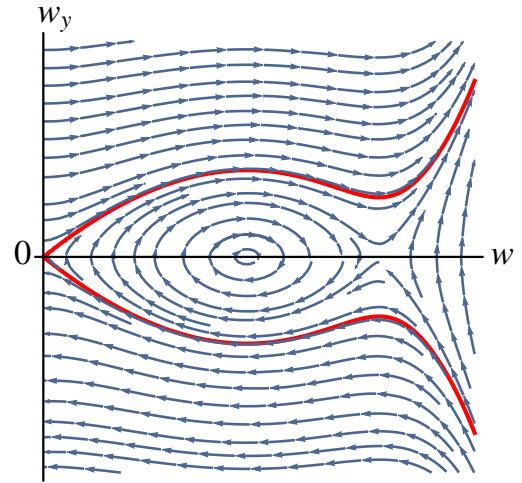

(c)

Figure 7: The phase plane of (4.17) for varying values of $\delta$. The level set $H_{f}=0$ is indicated in red. (a) $\delta=0.225<\delta_{\max }$, a planar homoclinic orbit exists. (b) $\delta=\delta_{\max } 4.22$, the planar homoclinic orbit deteriorates in a pair of heteroclinic orbits. (c) $\delta=0.235>\delta_{\max }$, no planar homoclinic orbit to the origin exists.

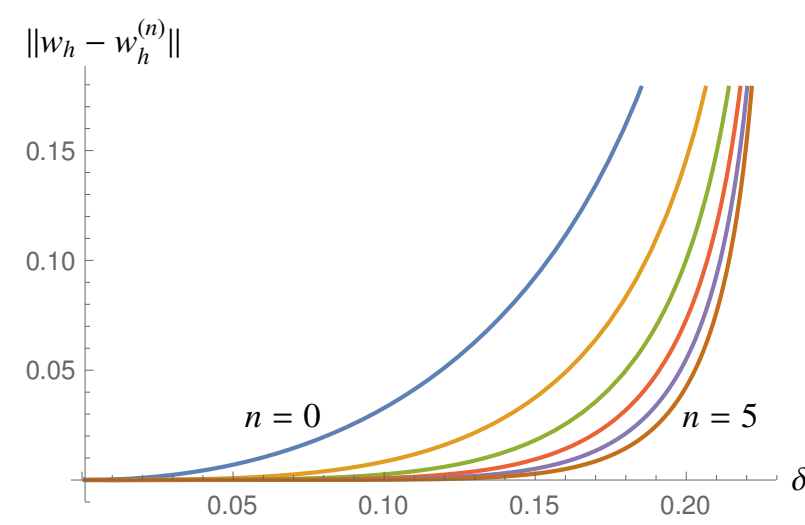

(a)

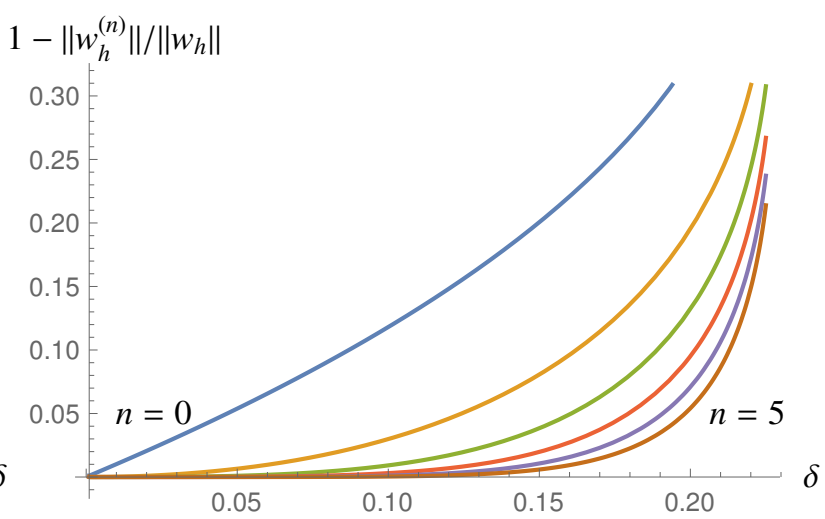

(b)

Figure 8: The approximation accuracy of the asymptotic series 4.23). (a) The $L^{2}$-norm of the difference of $w_{h}(y ; \delta)$, the unique homoclinic solution to 4.17) (computed numerically), and its $n$-th order asymptotic approximation $w_{h}^{(n)}(y ; \delta)$ according to 4.23 . (b) The relative error $1-\left\|w_{h}^{(n)}\right\| /\left\|w_{h}\right\|$.

See Figure 7 for an illustration of the dynamics of 4.17).

Note that, although the unique homoclinic solution $w_{h}(y ; \delta)$ to 4.17) does not have a closed-form expression, it can be approximated by substitution of an asymptotic expansion in $\delta$, as $0<\delta<\delta_{\max } \approx 0.23$ (4.22). Writing

$$
w_{h}(y ; \delta)=\delta \sum_{n=0}^{\infty} \delta^{n} \hat{w}_{n}(y),
$$

we find for the first terms

$$
\begin{aligned}
& \hat{w}_{0}(y)=\hat{w}_{1}(y)=\frac{3}{2} \operatorname{sech}^{2} \frac{y}{2}, \\
& \hat{w}_{2}(y)=\frac{3}{16}(4+13 \cosh y) \operatorname{sech}^{4} \frac{y}{2}
\end{aligned}
$$

see Figure 8 for a visualisation of the approximation accuracy of series 4.23.

\subsubsection{Slow dynamics}

As noted at its definition, $C_{0}$ (4.14) is a normally hyperbolic manifold that is invariant under the flow of (4.8). The flow on $C_{0}$ is given by

$$
\begin{aligned}
& u_{z}=\varepsilon_{1} p, \\
& p_{z}=-\varepsilon_{1} \varepsilon_{2} a\left(1-\varepsilon_{2} u\right) .
\end{aligned}
$$


bioRxiv preprint doi: https://doi.org/10.1101/2020.07.29.226522; this version posted June 24, 2021. The copyright holder for this preprint (which was not certified by peer review) is the author/funder. All rights reserved. No reuse allowed without permission.

This flow is linear; it has a unique saddle equilibrium at $(u, p)=\left(\varepsilon_{2}^{-1}, 0\right)$, with stable and unstable manifolds given by the stable and unstable linear subspaces

$$
\begin{aligned}
& E^{s}=\left\{(u, p) \in \mathbb{R}^{2} \mid p=\sqrt{a}\left(1-\varepsilon_{2} u\right)\right\}, \\
& E^{u}=\left\{(u, p) \in \mathbb{R}^{2} \mid p=-\sqrt{a}\left(1-\varepsilon_{2} u\right)\right\} .
\end{aligned}
$$

Note that the dynamics of (4.25) are slow in $z$; the eigenvalues of the saddle equilibrium are of order $O\left(\varepsilon_{1} \varepsilon_{2}\right)$.

\subsubsection{Constructing a stationary pulse solution}

We establish the existence of a stationary pulse solution to 4.1) by constructing a homoclinic orbit in system (4.8). This construction, which uses techniques and concepts from geometric singular perturbation theory, is based directly on the equivalent construction of pulses in the classical KGS model, as carried out in e.g. [13]. Due to the abundance of high quality sources, we choose to highlight central concepts in the pulse construction below. For detailed arguments and proofs, we refer to [12, 13, 14, 48].

The pulse solution that we want to construct, is an orbit that is homoclinic to the equilibrium $\left(\varepsilon_{2}^{-1}, 0,0,0,0\right)$ in (4.8). This equilibrium lies in particular on the invariant manifold $C_{0}$ (4.14). In order to construct a homoclinic orbit, we need to consider the flow both on $C_{0}$ and normal to $C_{0}$. The latter can be studied from a geometric viewpoint by considering the unstable and stable submanifolds of $C_{0}$ under the flow (4.8), denoted by $W^{u, s}\left(C_{0}\right)$, respectively. To leading order in $\varepsilon$ (and hence to leading order in $\varepsilon_{1,2}$ ), the flow normal to $C_{0}$ is given by the fast reduced system (4.15), as studied in subsection 4.1.1. The existence of a homoclinic orbit in this planar system implies that $W^{u}\left(C_{0}\right)$ and $W^{s}\left(C_{0}\right)$ intersect. Moreover, using the reversibility symmetry of (4.8) (i.e. the invariance of the flow (4.8) of under the reflection $(z ; p, q) \rightarrow(-z,-p,-q))$, one can show that this intersection is transversal; for details, see [13, 48]. Using the homoclinic solution $w_{h}(y ; \delta)$ to 4.17), we can track the fast flow normal to $C_{0}$ through this intersection. We define the interval

$$
I_{f}:=\left\{|z|<1 / \sqrt{\varepsilon_{1}}\right\},
$$

chosen such that $I_{f}$ is asymptotically large in $z$ but asymptotically small in $\varepsilon_{1} z$. We calculate the change of the slow variables $u$ and $p$ through the fast flow, over this interval, as follows. From (4.8), we see that $u_{z}$ is $O\left(\varepsilon_{1}\right)$, hence $u$ is constant to leading order in $z$; we write

$$
u=u_{0}+O\left(\varepsilon_{1}\right) .
$$

Moreover, $p_{z}$ is to leading order slaved to $u$ and $v$; we write

$$
p=p_{0}+\int_{0}^{z} u v^{2}+O\left(\varepsilon_{1} \varepsilon_{2}\right) \mathrm{d} \tilde{z}=p_{0}+u_{0} \int_{0}^{z} v^{2}+O\left(\varepsilon_{1}\right) \mathrm{d} \tilde{z} .
$$

We observe that, to leading order, $p-p_{0}$ is odd in $z$. The change of $u$ and $p$ over $I_{f}$ can now be calculated to leading order as

$$
\begin{aligned}
\Delta_{I_{f}} u & =\int_{I_{f}} u_{z} \mathrm{~d} z=\int_{I_{f}} \varepsilon_{1} p \mathrm{~d} z=2 p_{0} \sqrt{\varepsilon_{1}}+O\left(\varepsilon_{1}^{3 / 2}\right) \\
\Delta_{I_{f}} p & =\int_{I_{f}} p_{z} \mathrm{~d} z=\int_{I_{f}} u v^{2}+O\left(\varepsilon_{1} \varepsilon_{2}\right) \mathrm{d} z=u_{0} \int_{I_{f}} v^{2} \mathrm{~d} z+O\left(\varepsilon_{1}^{3 / 2}\right) \\
& =\frac{u_{0}}{h^{2} \sqrt{b}} \int_{-\infty}^{\infty} w_{h}(y ; \delta)^{2} \mathrm{~d} y+O\left(\varepsilon_{1}^{3 / 2}\right),
\end{aligned}
$$

cf. 4.16). Geometric singular perturbation theory [13, 14, 22, 30] enables us to construct an orbit by concatenating orbits on $C_{0}$ and normal to $C_{0}$; in particular, any orbit homoclinic to $\left(\varepsilon_{2}^{-1}, 0,0,0,0\right) \in C_{0}$ first flows away from this equilibrium along (and exponentially close to) $E^{u}$ 4.26. Then, it takes an excursion away from $C_{0}$, during which its evolution is to leading order determined by the fast reduced flow 4.15). After touching down again exponentially close to $C_{0}$, the $u$-component has not changed its value to leading order, but the $p$-component has, cf. (4.30). For the orbit to be biasymptotic to the equilibrium $\left(\varepsilon_{2}^{-1}, 0,0,0,0\right)$, this change needs to be such that the touchdown point lies exponentially close to $E^{s}(4.26$, such that the last, slow, orbit component takes us back to the $\left(\varepsilon_{2}^{-1}, 0,0,0,0\right)$ along $E^{s}$; see Figure 9 for a sketch of the geometric situation and construction.

For this concatenation construction to work, we find the condition that the 'vertical' $p$-distance between $E^{u}$ and $E^{s}$ must be equal to the leading order change in $p$ during the fast excursion over the interval $I_{f}$, as calculated in 4.30 , that is,

$$
2 \sqrt{a}=\frac{u_{0}}{h^{2} \sqrt{b}} \int_{-\infty}^{\infty} w_{h}(y ; \delta)^{2} \mathrm{~d} y .
$$




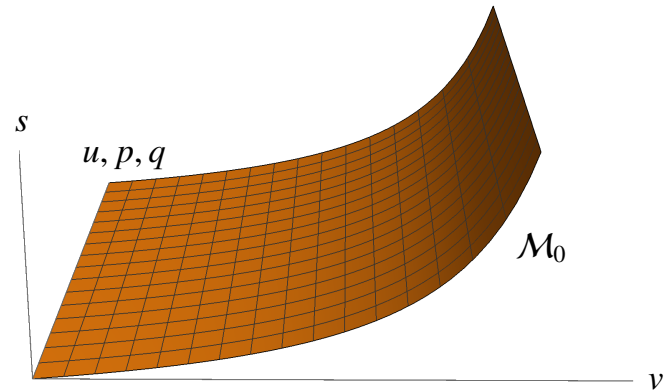

(a)

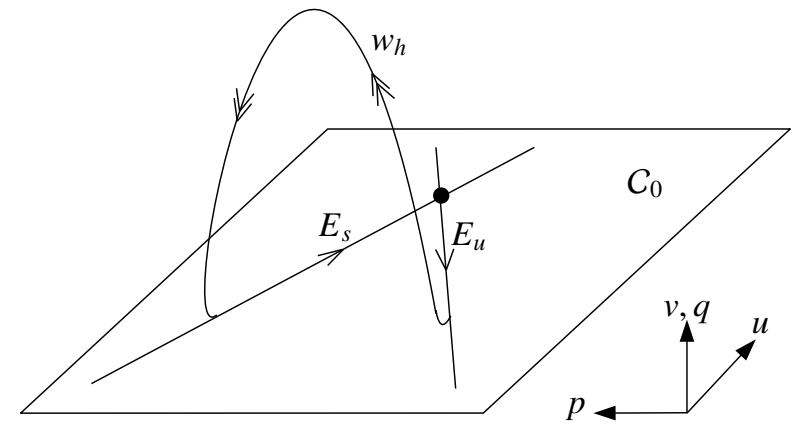

(b)

Figure 9: The geometric construction of the stationary pulse solution underlying Theorem 4.3 (a) The 4-dimensional invariant manifold $\mathcal{M}_{0}$ 4.12 embedded in 5-dimensional $(u, p, v, q, s)$-phase space. (b) On $\mathcal{M}_{0}$, the slow $(u, p)$-dynamics take place on the invariant manifold $C_{0}$ 4.14. The fast dynamics normal to $C_{0}$, can be used to connect the unstable and stable subspaces $E_{u}$ and $E_{s}$ (4.26) through the fast homoclinic orbit $w_{h}$ (see subsection 4.1.1; see also [13. Figures 2 and 3].

Using techniques from geometric singular perturbation theory, one can show that, for sufficiently small $\varepsilon_{1}$, condition (4.31) is not only necessary, but also sufficient to prove the existence of a homoclinic orbit in (4.8) that is asymptotically close to its singular concatenation. This existence follows from the persistence of $C_{0}$ and its stable and unstable manifolds $W^{u, s}\left(C_{0}\right)$, together with the observation that $W^{u}\left(C_{0}\right)$ and $W^{s}\left(C_{0}\right)$ intersect transversally; for more details and proofs, see [13, 14, 48]. For the purposes of this paper, it is sufficient to consider the outcome of previous equivalent analyses, namely that stationary pulse solutions are completely characterised by the existence condition 4.31, in the following way.

Theorem 4.3. Let $\varepsilon_{1}$ be sufficiently small, and let $u_{*}$ be a nondegenerate solution to

$$
2 \sqrt{a}=\frac{u_{*}}{h^{2} \sqrt{b}} \int_{-\infty}^{\infty} w_{h}\left(y ; b h / u_{*}\right)^{2} d y,
$$

where $w_{h}\left(y ; b h / u_{*}\right)$ is the unique, positive, nontrivial solution to 4.17) for which $\lim _{y \pm \infty} w_{h}\left(y ; b h / u_{*}\right)=0$. Then, system (4.8) admits an orbit that is homoclinic to the equilibrium $\left(\varepsilon_{2}^{-1}, 0,0,0,0\right)$. To leading order in $\varepsilon_{1}$, this orbit is given by

$$
\begin{aligned}
& u_{h}(z)=\varepsilon_{2}^{-1}-\left(\varepsilon_{2}^{-1}-u_{*}\right) e^{-\sqrt{a} \varepsilon_{1} \varepsilon_{2}|z|} \\
& v_{h}(z)=\frac{1}{h} w_{h}\left(\sqrt{b} z ; b h / u_{*}\right) \\
& s_{h}(z)=\frac{b v_{h}(z)}{1-h v_{h}(z)}
\end{aligned}
$$

Conversely, if (4.32) has no solution, then no orbit homoclinic to $\left(\varepsilon_{2}^{-1}, 0,0,0,0\right)$ exists.

Proof. The proof is completely analogous to that of [13, Theorem 4.1], with the fast planar homoclinic $w_{h}(y ; \delta)$ taking the role of $v_{0}[13$, equation (3.3)] therein. Since the arguments of the proof of [13, Theorem 4.1] depend only on the existence of such a planar homoclinic orbit, and not on its specific functional form, the reasoning in [13] holds ad verbatim for the construction described in section 4.1 which leads to the statement of Theorem 4.3

Remark 4.4 (Role of $\varepsilon_{2}$ ). Although (4.8) contains two asymptotically small, independent parameters $\varepsilon_{1}$ and $\varepsilon_{2}$, only $\varepsilon_{1}$ needs to be sufficiently small. The reason for this is that the time scale difference in 4.8 is only determined by $\varepsilon_{1}$ (4.27), and that the correction terms for the leading order expressions used in the geometric construction (e.g. (4.30) only depend on $\varepsilon_{1}$. The small parameter $\varepsilon_{2}$ solely measures the $u$-value of the homogeneous background state $\left(\varepsilon_{2}^{-1}, 0,0,0,0\right)$. Indeed, the analysis leading to Theorem 4.3 applies ad verbatim for the case $\varepsilon_{2}=1$; see also AppendixA

Note that condition 4.31) can be formulated in terms of $\delta$ as

$$
u_{*}=\frac{1}{2} \frac{b \sqrt{b}}{\sqrt{a}} \int_{-\infty}^{\infty} \frac{w_{h}(y ; \delta)^{2}}{\delta^{2}} \mathrm{~d} y
$$




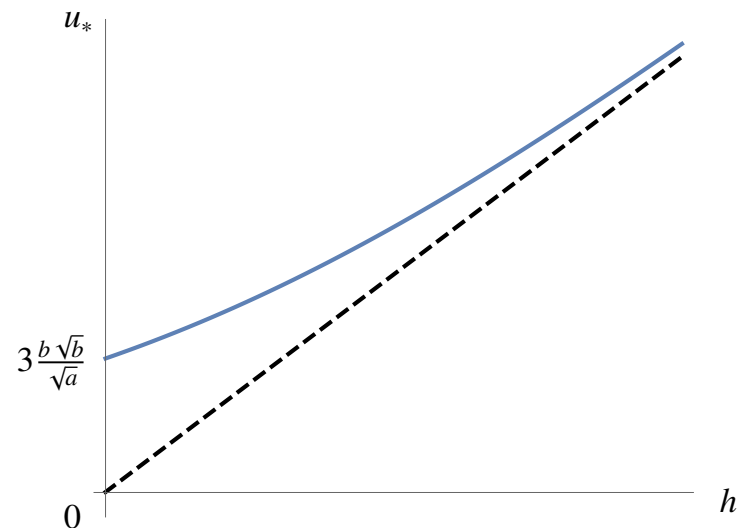

(a)

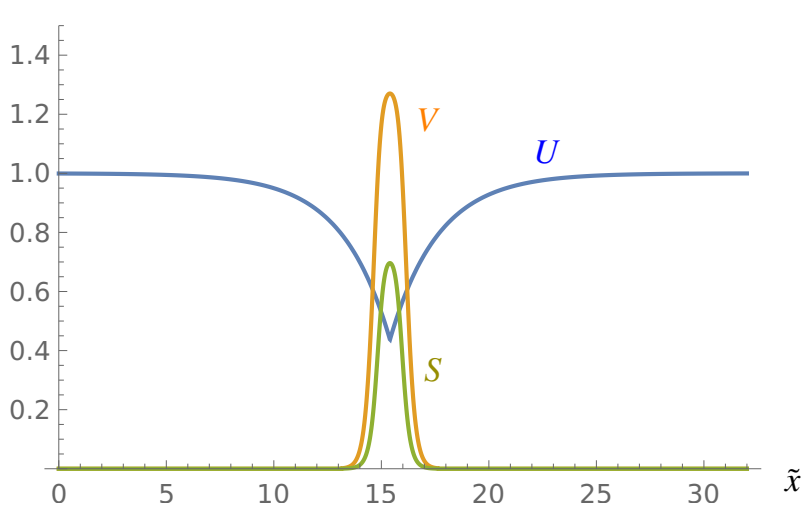

(b)

Figure 10: (a) The value of $u_{*}$, solving the existence condition 4.32 for increasing values of $h$ (with $a$ and $b$ fixed), in blue. The set of admissible values, for which $\delta=\frac{b h}{u_{*}}<\delta_{\max }$, is bounded below by the dashed line $u_{*}=\frac{b}{\delta_{\max }} h$. (b) A plot of the pulse solution established in Theorem 4.3 for the parameter values 3.4 , with $U$ in blue, $V$ in orange and $S$ in green.

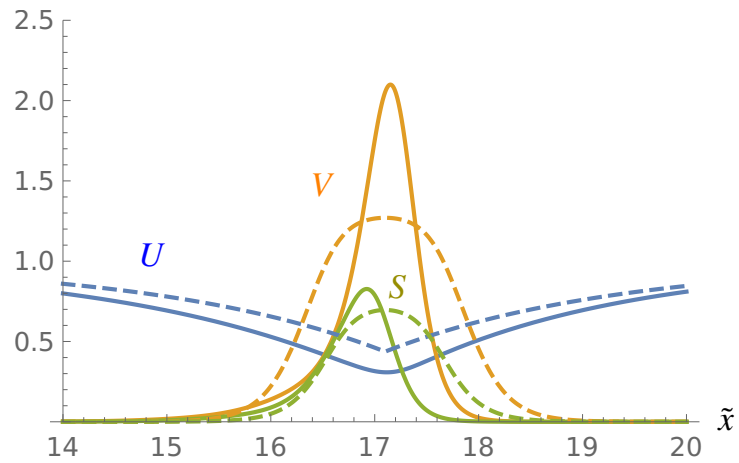

(a)

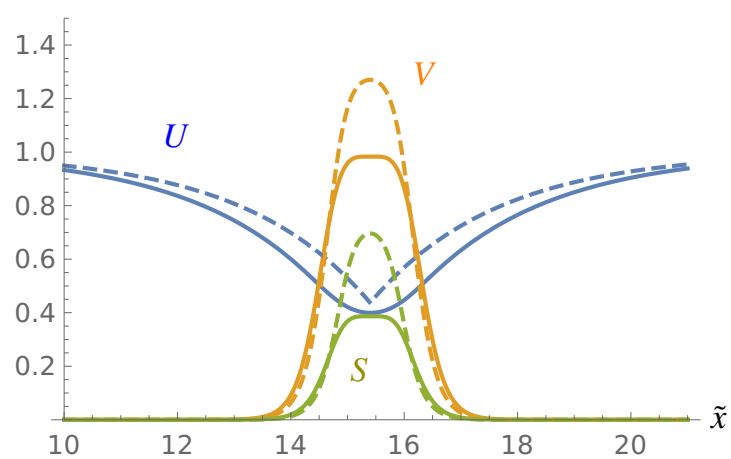

(b)

Figure 11: A comparison between direct numerical simulations of 4.1) (cf. Table 2, and the leading order analytical expression for a stationary pulse (see Theorem 4.3 and Figure 10 (b)), for parameter values $\mathcal{A}=\mathcal{B}=0.2, \mathcal{H}=0.5$ and $\varepsilon=0.1$, with $U$ in blue, $V$ in orange and $S$ in green. (a) The travelling pulse profile that is observed for $\mathcal{D}=4.5$, solid; the leading order analytical stationary pulse solution, dashed. The differences between the pulse profiles suggest that the numerically observed travelling pulse is not a straightforward perturbation of the analytical stationary pulse. (b) The stationary pulse profile that is observed for $\mathcal{D}=0.4$, solid; the leading order analytical stationary pulse solution, dashed. The difference between the pulse profiles are of $O(\varepsilon)$; the profiles are seen to match more closely when $\varepsilon$ is decreased.

taking the limit $h \rightarrow 0$, i.e. $\delta \rightarrow 0$ 4.17], yields

$$
u_{*}=\frac{1}{2} \frac{b \sqrt{b}}{\sqrt{a}} \int_{-\infty}^{\infty}\left(\frac{3}{2} \operatorname{sech}^{2} \frac{y}{2}\right)^{2} \mathrm{~d} y=3 \frac{b \sqrt{b}}{\sqrt{a}},
$$

cf. (4.23) and (4.24). This is in accordance with the observation that the limit $h \rightarrow 0$ of system (4.8) yields the classical KGS system, for which (4.35) has been derived as the existence condition of stationary pulse solutions [13, equation (4.2)]. See Figure 10 for the behaviour of $u_{*}$ for increasing values of $h$, and for a plot of the pulse solution given in Theorem 4.3

\subsection{Travelling pulses}

Pulses travelling with nonzero speed $C \neq 0$, i.e $c \neq 0$, are homoclinic orbits to system 4.77. In contrast to the stationary case studied in section 4.1, the dynamics of this system are fully 5 -dimensional. For clarity of presentation, we introduce the small parameter

$$
\varepsilon_{3}=\varepsilon^{\gamma-\frac{1}{2} \beta-1} ;
$$


note that $\varepsilon_{3}$ is indeed asymptotically small by conditions 4.6 . Using the previously defined small parameters $\varepsilon_{1,2}$ 4.9, we can rewrite system 4.7) as

$$
\begin{aligned}
u_{z} & =\varepsilon_{1} p, \\
p_{z} & =u v^{2}-\varepsilon_{1} \varepsilon_{2} a\left(1-\varepsilon_{2} u\right)-\varepsilon_{3} \varepsilon^{2} c p, \\
v_{z} & =q, \\
q_{z} & =b v-u v^{2}+h v s-\varepsilon_{3} c q, \\
c d \varepsilon_{3} \varepsilon^{\delta+\beta} s_{z} & =s-b v-h v s .
\end{aligned}
$$

This system can be treated in a similar fashion as in section 4.1. However, there are important differences. First, we observe that, unlike in the stationary situation (see section 4.1.1), the time scale of the dynamics of $(v, q, s)$ in system 4.37) is not immediately clear, because the asymptotic magnitude of $\varepsilon^{\delta+\beta}$ is not yet determined. In particular, depending on the sign and magnitude of $\delta+\beta$, the dynamics of $s$ may be faster, equivalent, or slower than those of $(v, q)$. We examine all three cases, and the subsequent construction of a travelling pulse, in subsections 4.2.2, 4.2.3 and 4.2.4 Secondly, it is important to note that that the invariant 2-dimensional hyperplane $C_{0}$ (4.14) that was defined in the context of stationary pulses, is also invariant under the flow of the 'nonzero speed system' 4.37). The dynamics of 4.37) on $C_{0}$ will be analysed in subsection 4.2.1.

\subsubsection{Slow dynamics}

On the invariant hyperplane $C_{0}$ (4.14), the dynamics of (4.37) are determined by the planar system

$$
\begin{aligned}
& u_{z}=\varepsilon_{1} p, \\
& p_{z}=-\varepsilon_{1} \varepsilon_{2} a\left(1-\varepsilon_{2} u\right)-\varepsilon_{3} \varepsilon^{2} c p .
\end{aligned}
$$

As in the stationary case (see subsection 4.1.2), the dynamics on $C_{0}$ are linear. Again, the only equilibrium is the saddle at $(u, p)=\left(\varepsilon_{2}^{-1}, 0\right)$. However, due to the advection term, the flow is not symmetric anymore with respect to reflection in the $u$-axis (compare (4.25) and (4.26); instead, the stable resp. unstable manifolds of the saddle equilibrium are given by the stable resp. unstable linear subspaces

$$
\begin{aligned}
& E^{s}=\left\{(u, p) \in \mathbb{R}^{2} \mid p=\left(1-\varepsilon_{2} u\right)\left(\frac{\varepsilon^{2} \varepsilon_{3} c}{2 \varepsilon_{1} \varepsilon_{2}}+\sqrt{a+\left(\frac{\varepsilon^{2} \varepsilon_{3} c}{2 \varepsilon_{1} \varepsilon_{2}}\right)^{2}}\right)\right\}, \\
& E^{u}=\left\{(u, p) \in \mathbb{R}^{2} \mid p=\left(1-\varepsilon_{2} u\right)\left(\frac{\varepsilon^{2} \varepsilon_{3} c}{2 \varepsilon_{1} \varepsilon_{2}}-\sqrt{a+\left(\frac{\varepsilon^{2} \varepsilon_{3} c}{2 \varepsilon_{1} \varepsilon_{2}}\right)^{2}}\right)\right\} .
\end{aligned}
$$

The dynamics of (4.38) are slow in $z$, as the eigenvalues of the saddle equilibrium are of order $O\left(\max \left(\varepsilon_{1} \varepsilon_{2}, \varepsilon_{3} \varepsilon^{2}\right)\right)$ - that is, asymptotically small.

\subsubsection{Constructing a travelling pulse solution, case I: s faster than $(v, q)$}

We assume that the $s$-dynamics are faster than the $(v, q)$-dynamics, that is, $\varepsilon_{3} \varepsilon^{\delta+\beta} \rightarrow 0$ as $\varepsilon \rightarrow 0$. Taking the limit $\varepsilon \rightarrow 0$ in (4.37) then yields

$$
\begin{aligned}
u_{z} & =0, \\
p_{z} & =u v^{2}, \\
v_{z} & =q, \\
q_{z} & =b v-u v^{2}+h v s, \\
0 & =s-b v-h v s .
\end{aligned}
$$

This is precisely the same system as studied in subsection 4.1.1. In particular, the same algebraic equation as (4.8e) defines the same 4-dimensional manifold $\mathcal{M}_{0}$ (4.12). Introducing the fast coordinate

$$
\zeta_{I}:=\frac{z}{\varepsilon_{3} \varepsilon^{\delta+\beta}}
$$


system 4.37) takes the form

$$
\begin{aligned}
u_{\zeta} & =\varepsilon_{1} \varepsilon_{3} \varepsilon^{\delta+\beta} p, \\
p_{\zeta} & =\varepsilon_{3} \varepsilon^{\delta+\beta}\left[u v^{2}-\varepsilon_{1} \varepsilon_{2} a\left(1-\varepsilon_{2} u\right)-\varepsilon_{3} \varepsilon^{2} c p\right], \\
v_{\zeta} & =\varepsilon_{3} \varepsilon^{\delta+\beta} q, \\
q_{\zeta} & =\varepsilon_{3} \varepsilon^{\delta+\beta}\left[b v-u v^{2}+h v s-\varepsilon_{3} c q\right], \\
c d s_{\zeta} & =s-b v-h v s,
\end{aligned}
$$

with singular limit

$$
\begin{aligned}
u_{\zeta} & =p_{\zeta}=v_{\zeta}=q_{\zeta}=0, \\
c d s_{\zeta} & =s-b v-h v s .
\end{aligned}
$$

It is easy to check using (4.43) that $\mathcal{M}_{0}$, which consists entirely of equilibria of the reduced fast system (4.43), is normally hyperbolic. Hence, by geometric singular perturbation theory (see e.g. [22, 30]), for sufficiently small $\varepsilon$, there exist a normally hyperbolic manifold $\mathcal{M}_{\varepsilon}$ that is invariant under the flow of the full 5-dimensional system 4.37; moreover, $\mathcal{M}_{\varepsilon}$ is $O\left(\varepsilon_{3} \varepsilon^{\delta+\beta}\right)$ close to $\mathcal{M}_{0}$.

In contrast to the stationary case (4.8), the dynamical system we are investigating here 4.42 is fully 5dimensional; hence, we need to look at the dynamics normal to $\mathcal{M}_{\varepsilon}$. Since these normal dynamics are onedimensional, $\mathcal{M}_{\varepsilon}$ is either uniformly attracting or uniformly repelling, depending on the sign of $c$. In either case, it follows that any bounded (in particular, any homoclinic) orbit of (4.37) must lie entirely on $\mathcal{M}_{\varepsilon}$. By the uniform asymptotic proximity of $\mathcal{M}_{\varepsilon}$ to $\mathcal{M}_{0}$, we can determine the dynamics on $\mathcal{M}_{\varepsilon}$ by a regular perturbation expansion of system 4.37) in powers of $\varepsilon_{3} \varepsilon^{\delta+\beta}$.

We also observe that, as $C_{0}$ is invariant under the full flow of (4.37), it necessarily holds that $C_{0} \subset \mathcal{M}_{\varepsilon}$. To construct a travelling pulse solution on $\mathcal{M}_{\varepsilon}$, we study the unstable and stable manifolds of $C_{0}$ in their intersection with $\mathcal{M}_{\varepsilon}$; depending on the sign of $c$, this effectively means we disregard the fast expansion $(c>0)$ or contraction $(c<0)$ in the (normal) $s$-direction. Defining the 3-dimensional manifolds

$$
\hat{W}^{u, s}\left(C_{0}\right):=W^{u, s}\left(C_{0}\right) \cap \mathcal{M}_{\varepsilon}
$$

we want to determine whether $\hat{W}^{u}\left(C_{0}\right)$ and $\hat{W}^{s}\left(C_{0}\right)$ intersect transversally, in order to repeat the construction procedure outlined in subsection 4.1, see also [12, 13, 14, 48]. From the existence of a planar homoclinic orbit in the fast singular limit 4.40) (see subsection 4.1.1), it follows that the singular limits of $\hat{W}^{u}\left(C_{0}\right)$ and $\hat{W}^{s}\left(C_{0}\right)$ coincide, as in the stationary case. However, due to the fact that the full system (4.37) does not exhibit reversibility symmetry due to the presence of the $c$-dependent terms, the question whether $\hat{W}^{u}\left(C_{0}\right)$ and $\hat{W}^{s}\left(C_{0}\right)$ intersect transversally for nonzero $\varepsilon$ cannot be answered based on symmetry arguments alone, as was the case in section 4.1.3, see also [13, 48]. Here, we need to perform a Melnikov calculation, along the lines of the analysis in [13, 48]. The Melnikov calculation is based on the observation that the singular limit system (4.40), in particular its planar reduction (4.15), is Hamiltonian. The associated conserved quantity $H_{f}(4.18$ ) is by definition zero along the singular planar homoclinic $w_{h}(y)$, as $w_{h}$ lies on the level set $H_{f}=0$; moreover, we see that $H_{f}$ identically vanishes on $C_{0}$. Hence, the total change of $H_{f}$ accumulated along any orbit in $\hat{W}^{u}\left(C_{0}\right) \cap \hat{W}^{s}\left(C_{0}\right)$ (which is necessarily biasymptotic to $C_{0}$ ), must vanish.

In terms of the original system variables $(u, p, v, q, s), H_{f}$ takes the form

$$
H_{f}(u, v, q)=\frac{h^{2}}{b} \frac{1}{2} q^{2}+h v+\log (1-h v)+\frac{h^{2}}{3 b} u v^{3} .
$$

Hence, the change of $H_{f}$ in $z$ is given by

$$
\begin{aligned}
\frac{\mathrm{d}}{\mathrm{d} z} H_{f} & =\frac{h^{2}}{b} q q_{z}+h v_{z}-\frac{h v_{z}}{1-h v}+\frac{h^{2}}{3 b} u_{z} v^{3}+\frac{h^{2}}{b} u v^{2} v_{z} \\
& =\frac{h^{2}}{b} q\left(b v-u v^{2}+h v s-\varepsilon_{3} c q\right)+h q-\frac{h q}{1-h v}+\frac{h^{2}}{3 b} \varepsilon_{1} p v^{3}+\frac{h^{2}}{b} u v^{2} q
\end{aligned}
$$

by 4.37). Using 4.37e, we can write

$$
h v s=\frac{b h v^{2}}{1-h v}+c d \varepsilon_{3} \varepsilon^{\delta+\beta} \frac{h v s_{z}}{1-h v},
$$


so that we obtain

$$
\frac{\mathrm{d}}{\mathrm{d} z} H_{f}=\frac{h^{2}}{b}\left[\varepsilon_{3} \varepsilon^{\delta+\beta} c d h \frac{v q s_{z}}{1-h v}-\varepsilon_{3} c q^{2}+\frac{1}{3} \varepsilon_{1} p v^{3}\right] .
$$

Following the analysis in [14, 48], we use the previously defined interval $I_{f}$ (4.27) to express the total change of $H_{f}$ accumulated along an orbit in $\hat{W}^{u}\left(C_{0}\right) \cap \hat{W}^{s}\left(C_{0}\right)$, as

$$
\Delta_{I_{f}} H_{f}=\int_{I_{f}} \frac{\mathrm{d}}{\mathrm{d} z} H_{f} \mathrm{~d} z=\frac{h^{2}}{b} \int_{I_{f}} \varepsilon_{3} \varepsilon^{\delta+\beta} c d h \frac{v q s_{z}}{1-h v}-\varepsilon_{3} c q^{2}+\frac{1}{3} \varepsilon_{1} p v^{3} \mathrm{~d} z .
$$

Now, we can use previous analysis on the reduced system (4.42) to obtain leading order expressions for $(v, q, s)$, which can be used to obtain a leading order expression for $\Delta_{I_{f}} H_{f}$. Defining the positive integrals

$$
\begin{aligned}
& \mathcal{I}_{1}(\delta):=\int_{-\infty}^{\infty} \frac{w_{h}}{\left(1-w_{h}\right)^{3}}\left(\frac{\mathrm{d} w_{h}}{\mathrm{~d} y}\right)^{2} \mathrm{~d} y, \\
& \mathcal{I}_{2}(\delta):=\int_{-\infty}^{\infty}\left(\frac{\mathrm{d} w_{h}}{\mathrm{~d} y}\right)^{2} \mathrm{~d} y, \\
& \mathcal{I}_{3}(\delta):=\int_{-\infty}^{\infty} w_{h}^{3} \mathrm{~d} y,
\end{aligned}
$$

we can use (4.10), 4.16, (4.28) and 4.29) to obtain the condition

$$
\varepsilon_{3} \varepsilon^{\delta+\beta} b c d \mathcal{I}_{1}(\delta)-\varepsilon_{3} c \mathcal{I}_{2}(\delta)+\frac{1}{3 b h} \varepsilon_{1} p_{0} \mathcal{I}_{3}(\delta)=0
$$

for $\Delta_{I_{f}} H_{f}$ to vanish to leading order. Note that the $h \rightarrow 0$ (i.e. $\delta \rightarrow 0$ ) limit of (4.51) can be calculated using the asymptotic expansion 4.23)-4.24, yielding

$$
\delta^{2}\left(-\varepsilon_{3} c \frac{6}{5}+\varepsilon_{1} \frac{p_{0}}{3 u_{0}} \frac{36}{5}\right)+O\left(\delta^{3}\right)=0,
$$

which is equivalent to [13, equation (3.14)] and [48, equation (2.24)].

As in the stationary case (cf. subsection 4.1.3), the second step in the construction of a pulse solution is to match the fast evolution through $\hat{W}^{u}\left(C_{0}\right) \cap \hat{W}^{s}\left(C_{0}\right)$ with the evolution on $\mathcal{C}_{0}$, such that the fast excursion normal to $C_{0}$ can be concatenated with the slow flow on $C_{0}$ along $E^{u, s}(4.39)$, in order to construct an orbit that is homoclinic to the equilibrium point $\left(\varepsilon_{2}^{-1}, 0,0,0,0\right) \in C_{0}$. To that end, we need to calculate the change of the slow componentents $(u, p)$ during the fast excursion. Since the dynamical equations for $u$ and $p$ in 4.37) differ from those in the stationary system (4.8) only by an asymptotically small term $\varepsilon_{3} \varepsilon^{2} c p$, and the leading order fast flow of (4.42) is the same as that of the stationary system (4.8), the leading order calculations 4.30) apply in the current setting as well. However, as the unstable and stable manifolds of the slow flow (4.39) differ from those in the stationary case (4.26), the resulting matching condition is different, and yields

$$
2 \sqrt{a+\left(\frac{\varepsilon^{2} \varepsilon_{3} c}{2 \varepsilon_{1} \varepsilon_{2}}\right)^{2}}=\frac{u_{0}}{h^{2} \sqrt{b}} \int_{-\infty}^{\infty} w_{h}(y ; \delta)^{2} \mathrm{~d} y
$$

Note that at this point, the asymptotic magnitude of the fraction $\frac{\varepsilon^{2} \varepsilon_{3}}{\varepsilon_{1} \varepsilon_{2}}$ is still undetermined. However, as the right hand side of the matching condition (4.53) is $O(1)$ in $\varepsilon$ and $\varepsilon_{1,2,3}$, the left hand side must be as well, hence it follows that either $\frac{\varepsilon^{2} \varepsilon_{3}}{\varepsilon_{1} \varepsilon_{2}}=1$ or $\frac{\varepsilon^{2} \varepsilon_{3}}{\varepsilon_{1} \varepsilon_{2}} \ll 1$.

Moreover, we can calculate the value of $p_{0}$ in the existence condition (4.51) using (4.29), by writing

$$
\begin{aligned}
2 p_{0} & =p_{0}+u_{0} \int_{0}^{\infty} v^{2}+O\left(\varepsilon_{1}\right) \mathrm{d} \tilde{z}+p_{0}-u_{0} \int_{-\infty}^{0} v^{2}+O\left(\varepsilon_{1}\right) \mathrm{d} \tilde{z} \\
& =\lim _{z \rightarrow \infty} p(z)+\lim _{z \rightarrow-\infty} p(z) \\
& =c \frac{\varepsilon^{2} \varepsilon_{3}}{\varepsilon_{1} \varepsilon_{2}}
\end{aligned}
$$

by (4.39); note that this is the same calculation as found in [48, equations (2.22-25)] and [13, equation (3.18)]. However, once we substitute this result into 4.51, we obtain the leading order existence condition

$$
\varepsilon_{3} c\left[\varepsilon^{\delta+\beta} b d \mathcal{I}_{1}(\delta)-\mathcal{I}_{2}(\delta)+\frac{1}{3 b h} \frac{\varepsilon^{2}}{2 \varepsilon_{2}} \mathcal{I}_{3}(\delta)\right]=0
$$




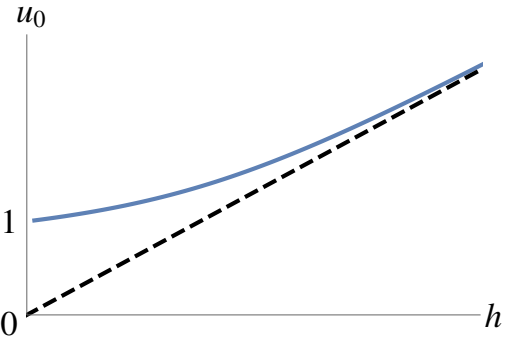

(a)

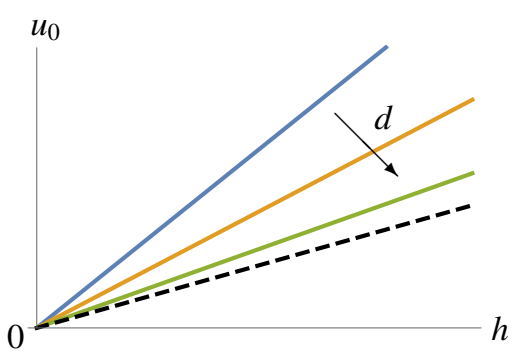

(b)

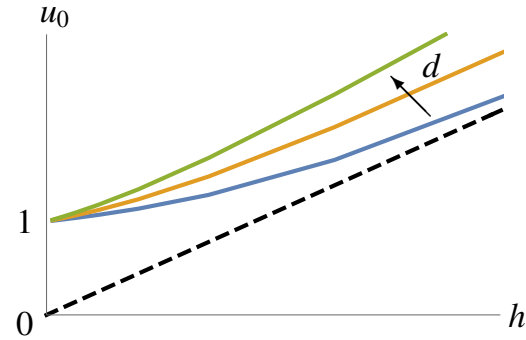

(c)

Figure 12: Solution curves of the existence condition 4.55, for different scaling choices of $\varepsilon^{\delta+\beta}$ and $\frac{\varepsilon^{2}}{\varepsilon_{2}}$, where $a$ and $b$ are fixed throughout. The set of admissible values, for which $\delta=\frac{b h}{u_{0}}<\delta_{\max }$, is bounded below by the dashed line $u_{0}=\frac{b h}{\delta_{\max }}$. (a) Dominant balance between the second $\left(I_{2}\right)$ and third $\left(I_{3}\right)$ term, i.e. $\frac{\varepsilon^{2}}{\varepsilon_{2}}=1$ and $\varepsilon^{\delta+\beta} \ll 1$. Here, $u_{0}$ is independent of $d$. (b) Dominant balance between the first $\left(I_{1}\right)$ and the second $\left(\mathcal{I}_{2}\right)$ term, i.e. $\frac{\varepsilon^{2}}{\varepsilon_{2}} \ll 1$ and $\varepsilon^{\delta+\beta}=1$. Here, $u_{0}$ is plotted as a function of $h$ for increasing values of $d$. (c) Equal balance between all three terms, i.e. both $\frac{\varepsilon^{2}}{\varepsilon_{2}}=1$ and $\varepsilon^{\delta+\beta}=1$. Again, $u_{0}$ is plotted as a function of $h$ for increasing values of $d$.

where we observe that $c$ occurs as a (nonzero) prefactor; hence, the leading order value of $c$ cannot be determined from 4.55). It follows that $c$ must be determined from 4.53); to be able to do this, we see that $\frac{\varepsilon^{2} \varepsilon_{3}}{\varepsilon_{1} \varepsilon_{2}}=1$, and thus $c$ is the solution of

$$
\frac{c^{2}}{4}=-a+\frac{1}{2}\left(\frac{u_{0}}{2 h^{2} \sqrt{b}} \int_{-\infty}^{\infty} w_{h}(y ; \delta)^{2} \mathrm{~d} y\right)^{2} .
$$

Moreover, to satisfy 4.55, we see that $\varepsilon^{\delta+\beta}$ or $\frac{\varepsilon^{2}}{\varepsilon_{2}}$ must be equal to one, since all integrals $\mathcal{I}_{1,2,3}$ are positive and at least one between the first $\left(\mathcal{I}_{1}\right)$ and the third $\left(\mathcal{I}_{3}\right)$ term must balance $\mathcal{I}_{2}$; from the same argument, it follows that neither term can be asymptotically large, hence $\varepsilon^{\delta+\beta} \leq 1$ and $\frac{\varepsilon^{2}}{\varepsilon_{2}} \leq 1$. Thus, three distinct situations arise, where only the first $\left(\mathcal{I}_{1}\right)$ term, only the third $\left(\mathcal{I}_{3}\right)$ term, or both the first and the third term balance the second $\left(\mathcal{I}_{2}\right)$ term in the existence condition (4.55) In Figure 12, several parameter-dependent solution curves for these three different scaling choices of $\varepsilon^{\delta+\beta}$ and $\frac{\varepsilon}{\varepsilon_{2}}$ are shown.

We summarise the findings of this subsection in the following theorem:

Theorem 4.5. Let $\varepsilon$ be sufficiently small. Moreover, let $\gamma=1+\frac{3}{2} \beta-\kappa$, and assume at least one of the following conditions holds:

$$
\begin{aligned}
& 2-\kappa=0 \quad \text { and } \quad \delta+\beta \geq 0 \\
& 2-\kappa \geq 0 \quad \text { and } \quad \delta+\beta=0 .
\end{aligned}
$$

Then, system (4.37) admits a homoclinic orbit to the equilibrium $\left(\varepsilon_{2}^{-1}, 0,0,0,0\right)$, provided equation (4.56) admits a real solution for $c$, where $\delta$ is chosen such that condition (4.55) is satisfied to leading order in $\varepsilon$.

The homoclinic orbit is, to leading order in $\varepsilon$, given by

$$
\begin{aligned}
& u_{h}(z)=\varepsilon_{2}^{-1}-\left(\varepsilon_{2}^{-1}-u_{0}\right) e^{-\frac{c}{2} \varepsilon_{1} \varepsilon_{2} z} e^{-\varepsilon_{1} \varepsilon_{2}|z| \sqrt{a+\frac{c^{2}}{4}}}, \\
& v_{h}(z)=\frac{1}{h} w_{h}\left(\sqrt{b} z ; b h / u_{0}\right), \\
& s_{h}(z)=\frac{b v_{h}(z)}{1-h v_{h}(z)} .
\end{aligned}
$$

Conversely, if either (4.56) has no solution for real c, or (4.55) cannot be satisfied, then no orbit homoclinic to $\left(\varepsilon_{2}^{-1}, 0,0,0,0\right)$ exists.

Remark 4.6 (Non-existence of travelling pulses in Gray-Scott). The existence result of Theorem 4.5 does not contradict the non-existence result [13, Theorem 5.1] for travelling pulses, as the scaling regimes 4.57a], 4.57b were not considered in [13]; see also [12, Remark 4.1].

Proof. For sufficiently small $\varepsilon_{3} \varepsilon^{\delta+\beta}$, Fenichel's invariant manifold theorem [22, 30] provides the existence of $\mathcal{M}_{\varepsilon}$, which is invariant under the flow of (4.37) and $O\left(\varepsilon_{3} \varepsilon^{\delta+\beta}\right)$ close to $\mathcal{M}_{0}$ (4.12). Specifically, there exists $\varepsilon_{0, A}>0$ such that for all $0<\varepsilon<\varepsilon_{0, A}$, the persistence of $\mathcal{M}_{\varepsilon}$ as an invariant manifold is guaranteed. On this 4-dimensional invariant manifold $\mathcal{M}_{\varepsilon}$, the transversal intersection of the restricted unstable and stable manifolds of $C_{0}$ (4.44), $\hat{W}^{u}\left(C_{0}\right) \cap \hat{W}^{s}\left(C_{0}\right)$, follows from leading order calculations presented in subsection 4.2 .2 combined with geometric arguments from the proof of [13, Theorem 4.1] and/or [48, Theorem 2.1]. The latter only needs to be augmented 
bioRxiv preprint doi: https://doi.org/10.1101/2020.07.29.226522; this version posted June 24, 2021. The copyright holder for this preprint (which was not certified by peer review) is the author/funder. All rights reserved. No reuse allowed without permission.

in the construction of the take-off and touchdown curves, where the intersection with the hyperplane $\{q=0\}$ now needs to be considered in its intersection with $\mathcal{M}_{\varepsilon}$. Geometric perturbation theory now provides the existence of $\varepsilon_{0, B}>0$ such that for all $0<\varepsilon<\varepsilon_{0, B}$, a homoclinic orbit to $\left(\varepsilon_{2}^{-1}, 0,0,0,0\right)$ exists within $\mathcal{M}_{\varepsilon}$; defining the global upper $\varepsilon$-bound $\hat{\varepsilon}_{0}:=\min \left(\varepsilon_{0, A}, \varepsilon_{0, B}\right)$ completes the proof.

It is useful to explore the ramifications of the scaling choices made in this subsection for the original system 4.1). As mentioned in theorem 4.5 the condition $\frac{\varepsilon^{2} \varepsilon_{3}}{\varepsilon_{1} \varepsilon_{2}}=1$ fixes $\gamma=1+\frac{3}{2} \beta-\kappa$, which, in combination with condition (4.6), implies $\beta-\kappa>0$.

The scaling choice (4.57a), which balances $\mathcal{I}_{2}$ with $\mathcal{I}_{3}$, implies $\beta>2$. Taken together with $\kappa=2$, we find for the scaling exponents of the original model parameters 4.5 :

$$
\begin{gathered}
\mathcal{A}=o\left(\varepsilon^{4}\right), \mathcal{B}=o\left(\varepsilon^{2}\right), C=o\left(\varepsilon^{2}\right), \frac{1}{\mathcal{H}}=o(1), \\
U=O\left(\varepsilon^{2}\right), V=o(1), S=o\left(\varepsilon^{2}\right), \frac{1}{\tilde{z}}=o(1),
\end{gathered}
$$

while the sign of $\delta$, the scaling exponent of $\mathcal{D}$, is not fixed. In particular, we see that $\mathcal{A}$ scales with a relatively high power of $\varepsilon$; hence, a travelling pulse whose existence is established by theorem 4.5 with scaling (4.57a), can only be observed for very small values of $\mathcal{A}$. In addition, the $h \rightarrow 0$ limit of 4.55 ) yields $u_{0}=1$ and $\frac{1}{2} c^{2}=-a+\frac{9}{2} b^{3}$ (see also Figure 12 (a)). That is, in the classical KGS limit, the amplitude of the pulse does, to leading order, not depend on the system parameters; this is in clear contrast to the stationary pulses studied in this paper (see Theorem 4.3) and to the travelling pulses constructed in [48].

On the other hand, the scaling choice 4.57a), which balances $\mathcal{I}_{2}$ with $\mathcal{I}_{1}$, only implies $\beta>0$. Taken together with $0<\kappa \leq 2$, we find for the scaling exponents of the original model parameters 4.5 :

$$
\begin{aligned}
\mathcal{A}=o\left(\varepsilon^{2}\right), \mathcal{B} & =o(1), C=o(\varepsilon), \frac{1}{\mathcal{D}}=o(1), \frac{1}{\mathcal{H}}=o(1), \\
U & =o(1), V=o(1), S=o(1), \frac{1}{\tilde{z}}=o\left(\varepsilon^{-1}\right) .
\end{aligned}
$$

Compared with 4.59 , the conditions on the scaling components are rather mild. Still, the condition $\beta-\kappa>0$ implies that $O(\mathcal{A} / \mathcal{B})=o\left(\varepsilon^{2}\right)$ - that is, $\mathcal{A}$ and $\mathcal{B}$ differ at least by two orders of magnitude in $\varepsilon$. Note that the $h \rightarrow 0$ limit of 4.55 yields $u_{0}=0$ (see also Figure 12 (b)), accentuating the fact that this solution branch does not exist in the classical KGS system.

\subsubsection{Constructing a travelling pulse solution, case II: $(v, q, s)$-dynamics on the same scale}

We assume that the $s$-dynamics occur on the same scale as the $(v, q)$-dynamics, that is, $\varepsilon_{3} \varepsilon^{\delta+\beta}=1$. Taking the limit $\varepsilon \rightarrow 0$ in 4.37 now yields

$$
\begin{aligned}
u_{z} & =0, \\
p_{z} & =u v^{2}, \\
v_{z} & =q, \\
q_{z} & =b v-u v^{2}+h v s, \\
c d s_{z} & =s-b v-h v s .
\end{aligned}
$$

The construction method of the previous sections can be applied if and only if the reduced system (4.61) admits a homoclinic orbit. In contrast to the previous cases (cf. (4.15) and (4.40), where the effective phase space was planar, the dynamics of (4.61) are effectively three-dimensional. This poses a considerate analytical challenge, as the dynamics of nonlinear three-dimensional dynamical systems can be highly complex. That being said, it seems sensible to start from a situation where the existence of a homoclinic orbit is known - that is, in the singular limit $c d=0$. In this limit, the analysis of section 4.1.1 applies, and provides us with an homoclinic orbit $\left(v_{h}(z), q_{h}(z), s_{h}(z)\right)$. Now, we can use Melnikov theory [24, section 2.1] to determine whether the intersection of the stable and unstable manifolds of the origin $(v, q, s)=(0,0,0)$ persists for $c d>0$. To that end, we use the conserved quantity $H_{f}$ in terms of the original variables $(v, q)$ 4.46. However, we find that

$$
\int_{-\infty}^{\infty} \frac{\mathrm{d}}{\mathrm{d} z} H_{f} \mathrm{~d} z=\int_{-\infty}^{\infty} c d \frac{h^{3}}{b} \frac{v q s_{z}}{1-h v} \mathrm{~d} z=c d h^{3} \int_{-\infty}^{\infty} \frac{v_{h}}{\left(1-h v_{h}\right)^{3}}\left(\frac{\mathrm{d} v_{h}}{\mathrm{~d} z}\right)^{2} \mathrm{~d} z+O\left((c d)^{2}\right)
$$

is always positive, since $v_{h}>0$ by assumption, and $1-h v_{h}>0$, see (4.11). This suffices to conclude that the intersection for $c d=0$ does not persist for small positive values of $c d$. 
bioRxiv preprint doi: https://doi.org/10.1101/2020.07.29.226522; this version posted June 24,2021 . The copyright holder for this preprint (which was not certified by peer review) is the author/funder. All rights reserved. No reuse allowed without permission.

While, in principle, the existence of homoclinic orbits in 4.61) for non-small values of $c d$ is not ruled out by the above argument, their analytical inaccessibility makes the situation created by the scaling choice considered in this section, fall outside the scope of this paper. Hence, we disregard the (limiting) case $\varepsilon_{3} \varepsilon^{\delta+\beta}=1$.

\subsubsection{Constructing a travelling pulse solution, case III: $s$ slower than $(v, q)$}

We assume that the $s$-dynamics are slower than the $(v, q)$-dynamics, that is, $\frac{1}{\varepsilon_{3} \varepsilon^{\delta+\beta}} \rightarrow 0$ as $\varepsilon \rightarrow 0$. Multiplying 4.37e with $\frac{1}{\varepsilon_{3} \varepsilon^{\delta+\beta}}$ and subsequently taking the limit $\varepsilon \rightarrow 0$ in 4.37 then yields

$$
\begin{aligned}
u_{z} & =0, \\
p_{z} & =u v^{2}, \\
v_{z} & =q, \\
q_{z} & =b v-u v^{2}+h v s, \\
c d s_{z} & =0 .
\end{aligned}
$$

The existence of a homoclinic orbit in 4.63) now follows directly from the analysis in [13, 48], yielding

$$
v_{h}=\frac{3}{2} \frac{b+h s_{0}}{u_{0}} \operatorname{sech}^{2} \frac{\sqrt{b+h s_{0}}}{2} z .
$$

In principle, one could mimic the analytical steps taken in subsection 4.2.2, to obtain existence conditions along the lines of (4.55), which would depend on $s_{0}$. However, for reasons that will become clear below, we will consider the dynamics of the slower variables $(u, p, s)$ first.

The set of equilibria of the reduced system 4.63 is given by the 3-dimensional hyperplane

$$
C_{1}:=\left\{(u, p, v, q, s) \in \mathbb{R}^{5} \mid v=0, q=0\right\} .
$$

Like $C_{0}$ (4.14), $C_{1}$ is invariant under the flow of the full 5-dimensional system (4.37). The (u,p)-dynamics on $C_{1}$ are the same as those on $C_{0}$, and are therefore given by system 4.25. However, the $s$-dynamics on $C_{1}$ are given by

$$
c d \varepsilon_{3} \varepsilon^{\delta+\beta} s_{z}=s
$$

which are unbounded for $z \rightarrow \infty$. Hence, we can conclude that in the scaling chosen in this subsection, any orbit homoclinic to $\left(\varepsilon_{2}^{-1}, 0,0,0,0\right)$ in 4.37 , being necessarily bounded, must have a trivial $s$-component - that is, must be a solution to the classical KGS system.

\section{Conclusion and outlook}

The aim of this paper is to analytically investigate the influence of autotoxicity on vegetation patterns, through the analysis of travelling pulse solutions to the biomass-water-toxicity model (2.1). In particular, we want to analytically explain the occurence of travelling, asymmetric pulses, as observed in numerical simulations of the biomass-water-toxicity system, see e.g. Figure 3 . The numerical evidence presented in section 3 suggests that the presence of toxicity is a prerequisite for the occurence of these travelling pulses in particular (compare Figures 2 and 3), and for previously unobserved dynamic spatio-temporal patterns in general (cf. Figure 1).

However, while the inclusion of an additional model component to the classical KGS model enriches the class of spatial patterns produced by this model with a number of provocative spatiotemporal patterning phenomena, it also significantly increases the mathematical complexity of the model, and subsequently of its analysis. Where the occurence of several types of patterns in the classical KGS model sensitively depends on the asymptotic scaling of the model components and parameters (see e.g. [12, Figure 2]), this is even more so the case in the extended model (2.4) considered in this paper. Therefore, the main emphasis of the analytical part of this paper (section 4) is on the balance of asymptotic scalings. The result of this analysis is that, within the scaling preparatory scaling choices made in AppendixA, stationary and travelling pulses can only be constructed in specific asymptotic scalings. This 'positive' outcome, as summarised in Theorems 4.3 and 4.5 , is balanced by the 'negative' outcome of subsections 4.2 .3 and 4.2 .4

Comparing the statement of Theorem 4.5 and its ramifications for the parameter scalings in the nondimensionalised model (2.4), it is clear that the travelling pulses constructed in subsection 4.2.2 are not the travelling pulses observed in numerical simulations, shown in Figure 3. In particular, the numerically observed pulses vanish when the parameters $\mathcal{A}$ and $\mathcal{B}$ are not approximately equal (see Table 2), whereas the travelling pulses from Theorem 4.5 only exist when $\mathcal{A}$ and $\mathcal{B}$ differ by two orders of magnitude in $\varepsilon$ (cf. (4.59) and (4.60). This discrepancy between analytical results and numerical observations could be explained in several ways. 
bioRxiv preprint doi: https://doi.org/10.1101/2020.07.29.226522; this version posted June 24, 2021. The copyright holder for this preprint (which was not certified by peer review) is the author/funder. All rights reserved. No reuse allowed without permission.

- Stability properties. As mentioned in section 3, our numerical investigation indicates that the travelling pulse observed in the simulations for specific parameter ranges is stable in the PDE sense. Since the travelling pulses constructed analytically in section 4 are not recoverable in our simulations - i.e., even when the parameter constraints in (4.59), 4.60) are obeyed and/or we consider the leading order approximation of the pulse in (4.58) as initial condition the system converges to a uniform steady state and the patterned structure breaks down - we expect the analytically constructed pulses to be unstable in the PDE sense.

- Boundary effects. The numerical simulations have been carried out on a bounded domain, whereas the analytical results assume an unbounded spatial domain. Hence, the existence of travelling pulses could be accredited to boundary effects - in particular, to self-interaction through the boundary. This would mean that a single travelling pulse on an unbounded domain is not the proper mathematical abstraction of the numerical observations as in Figure 3 Rather, one should consider a pair of interacting pulses, along the lines of [12]. However, our numerical investigations reveal that the observed travelling pulse solutions persist (both shape and speed) when doubling of the domain length, which is not in line with the hypothesis of self-interaction (see Remark 3.1).

- Transient behaviour. The numerical simulations have been carried out for a finite time, whereas the existence analysis is time-independent. Hence, the observed travelling pulses could be the manifestation of a slow transient process from initial state to bare soil, where these travelling pulses only exist for a finite time. This would mean that a time-independent travelling pulse, that is, a stationary pulse in a co-moving frame with fixed speed, is not the proper mathematical abstraction of the numerical observations as in Figure 3. However, our numerical investigations reveal that the observed travelling pulse solutions persist when doubling the simulation time (see Remark 3.2). This does not rule out the hypothesis of metastability, but it does severely limit the evolution speed of the wave profile. Transitional pattern formation phenomena have be studied analytically, see e.g. [3]; one could adopt the approach outlined in [3] to study (2.4). However, the absence of integrable structure might prohibit this approach.

- Scaling assumptions. As outlined in the introduction of section 4 the applicability of the geometric singular perturbation theory approach to pulse patterns, as used in [12, 13, 14, 48], is closely tied to the asymptotic scaling of the underlying model (2.4). The arguments leading to the preparatory scaling choices (4.5) are specified in AppendixA However, it is important to note that not every scaling choice made in AppendixA is necessary for the application of the geometric construction techniques. The two scaling choices pertaining to the dynamics of the toxicity component $s(\mathrm{~A} .17 \mathrm{p})$ and $\mathrm{A} .20 \mathrm{p})$ are convenient, rather than necessary. In particular, the assumption underlying the last scaling choice $A .20$ - namely, that the stationary pulse constructed in section 4.1 should be the ' $c=0$ ' member of a family of travelling (' $c \neq 0$ ') pulses - could prove to be too restrictive. After all, families of travelling waves that do not include a neighbourhood of $c=0$ regularly occur [8, 51, 53]. In addition, the 'proper' scaling needed to understand the travelling pulse shown in Figure 5 need not be uniform: abandoning scalings (A.17) and (A.20) might lead to the situation where the nonlinear interaction terms govern the singular behaviour of the pulse solution. In such a case, one would need to use geometric blowup techniques to construct a singular concatenated homoclinic orbit, along the lines of [25].

The analysis of travelling pulses incorporating this generalised scaling is ongoing work.

It is important to note that, while the analytical approach advocated in this paper applies to the existence of stationary and travelling pulse solutions, the question of pulse stability is still very much open. We plan to apply the techniques developed for the stability analysis of pulses in two-component reaction-diffusion systems, as presented in [14], to the three-component reaction-diffusion-ODE system (2.4). The method presented in [14], which is based on an Evans function approach, does not in principle depend on the number of model components, and can be applied to pattern solutions in $n$-dimensional reaction-diffusion systems [47]. Moreover, this approach is amenable to be extended to systems of mixed (reaction-diffusion-ODE) type. The stability of pulse solutions to the biomass-water-toxicity model 2.1) will be investigated in a separate work.

Moreover, numerical simulations in [35] on two-dimensional domains reveal the presence of both crescent (travelling) moon spots and double-scale patterns for some parameter regimes. In these double-scale pattens, the pulses/fronts travel at a micro-scale level within a pattern, which appears stable on a macroscopic scale. Such multiple-scale behaviour has been connected with pattern robustness in a different, but related, ecological context [33]. Future challenges hence involve the analytical investigation of such structures - in the first case (travelling crescent moons), an approach along the lines of [21] would be a prime candidate.

The biomass-water-toxicity model by Marasco et al. (2.1) has proven to be a rich and inspiring source of previously unobserved patterning phenomena. It is important to emphasise that autotoxicity can be used to explain and 
bioRxiv preprint doi: https://doi.org/10.1101/2020.07.29.226522; this version posted June 24,2021 . The copyright holder for this preprint (which was not certified by peer review) is the author/funder. All rights reserved. No reuse allowed without permission.

recreate experimentally observed dynamical patterns, without having to assume a specific domain topography, in contrast to previous work [1, 2, 52]. In this respect, the model is interesting both from an ecological perspective, and from the more general viewpoint of mathematical modelling. Systems of reaction-diffusion-ODE type have been the subject of recent investigations; in particular, the shape and stability properties of patterns have proven to be significantly different from their 'classical reaction-diffusion' counterparts, sometimes leading to counterintuitive results [36, 37]. We hope that the work presented in this paper, though exploratory, will lead to a deeper understanding and a broader appreciation of systems of this type, of which the biomass-water-toxicity model (2.1) is an intriguing example.

\section{Acknowledgements}

AI acknowledges partial support from the New Frontier's grant NST-0001 of the Austrian Academy of Sciences ÖAW and an FWF Hertha Firnberg Research Fellowship (T 1199-N). FV was supported by a Humboldt Research Fellowship.

\section{Competing interests statement}

The authors state that there is no conflict of interests.

[1] R. Bastiaansen, P. Carter, and A. Doelman. Stable planar vegetation stripe patterns on sloped terrain in dryland ecosystems. Nonlinearity 32(8): pp. 2759-2814 (2019). doi:10.1088/1361-6544. URL https://doi.org/10.1088/1361-6544

[2] R. Bastiaansen and A. Doelman. The dynamics of disappearing pulses in a singularly perturbed reactiondiffusion system with parameters that vary in time and space. Physica D: Nonlinear Phenomena 388: pp. 45-72 (2019). doi:10.1016/j.physd.2018.09.003. URL https://doi.org/10.1016/j.physd.2018.09.003

[3] M. Beck and C. Eugene Wayne. Using global invariant manifolds to understand metastability in the Burgers equation with small viscosity. SIAM Review 53(1): pp. 129-153 (2011). doi:10.1137/100808836. URL https://doi .org/10.1137/100808836

[4] G. Bonanomi, G. Incerti, E. Barile, M. Capodilupo, V. Antignani, A. Mingo, V. Lanzotti, F. Scala, and S. Mazzoleni. Phytotoxicity, not nitrogen immobilization, explains plant litter inhibitory effects: evidence from solid-state 13c NMR spectroscopy. New Phytologist 191(4): pp. 1018-1030 (2011). doi:10.1111/j. 1469-8137.2011.03765.x. URL https://doi.org/10.1111\%2Fj .1469-8137.2011.03765.x

[5] G. Bonanomi, G. Incerti, A. Stinca, F. Cartenì, F. Giannino, and S. Mazzoleni. Ring formation in clonal plants. Community Ecology 15(1): pp. 77-86 (2014). doi:10.1556/comec.15.2014.1.8. URL https://doi.org/10.1556\%2Fcomec.15.2014.1.8

[6] F. Cartenì, A. Marasco, G. Bonanomi, S. Mazzoleni, M. Rietkerk, and F. Giannino. Negative plant soil feedback explaining ring formation in clonal plants. Journal of Theoretical Biology 313: pp. 153-161 (2012). doi:10.1016/j.jtbi.2012.08.008. URL https://doi.org/10.1016\%2Fj.jtbi.2012.08.008

[7] W. Chen and M. Ward. The stability and dynamics of localized spot patterns in the two-dimensional Gray-Scott model. SIAM Journal on Applied Dynamical Systems 10(2): pp. 582-666 (2011). doi: 10.1137/09077357X. URL https://doi.org/10.1137/09077357X

[8] X. Chen and Y. Qi. Sharp estimates on minimum travelling wave speed of reaction diffusion systems modelling autocatalysis. SIAM Journal on Mathematical Analysis 39(2): pp. 437-448 (2007). doi: $10.1137 / 060665749$. URL https://doi.org/10.1137/060665749

[9] V. Deblauwe, N. Barbier, P. Couteron, O. Lejeune, and J. Bogaert. The global biogeography of semi-arid periodic vegetation patterns. Global Ecology and Biogeography 17(6): pp. 715-723 (2008). doi:10.1111/j. 1466-8238.2008.00413.x. URL https://doi.org/10.1111\%2F j .1466-8238.2008.00413.x 
bioRxiv preprint doi: https://doi.org/10.1101/2020.07.29.226522; this version posted June 24, 2021. The copyright holder for this preprint (which was not certified by peer review) is the author/funder. All rights reserved. No reuse allowed without permission.

[10] V. Deblauwe, P. Couteron, J. Bogaert, and N. Barbier. Determinants and dynamics of banded vegetation pattern migration in arid climates. Ecological Monographs 82(1): pp. 3-21 (2012). doi:10.1890/11-0362.1. URL https://doi .org/10.1890\%2F11-0362.1

[11] S. Dekker, H. de Boer, V. Brovkin, K. Fraedrich, M. Wassen, and M. Rietkerk. Biogeophysical feedbacks trigger shifts in the modelled vegetation-atmosphere system at multiple scales. Biogeosciences 7(4): pp. 1237-1245 (2010). doi:10.5194/bg-7-1237-2010. URL https://doi .org/10.5194/bg-7-1237-2010

[12] A. Doelman, W. Eckhaus, and T. Kaper. Slowly modulated two-pulse solutions in the Gray-Scott model II: geometric theory, bifurcations, and splitting dynamics. SIAM Journal on Applied Mathematics 61(6): pp. 2036-2062 (2001). doi:10.1137/S0036139900372429. URL https://doi.org/10.1137/S0036139900372429

[13] A. Doelman, T. Kaper, and P. Zegeling. Pattern formation in the one-dimensional Gray-Scott model. Nonlinearity 10(2): pp. 523-563 (1997). doi:10.1088/0951-7715/10/2/013. URL https://doi .org/10.1088/0951-7715/10/2/013

[14] A. Doelman and F. Veerman. An explicit theory for pulses in two component, singularly perturbed, reactiondiffusion equations. Journal of Dynamics and Differential Equations 27(3): pp. 555-595 (2015). doi:10. 1007/s10884-013-9325-2. URL https://doi.org/10.1007/s10884-013-9325-2

[15] M. Eppinga, M. Baudena, D. Johnson, J. Jiang, K. Mack, A. Strand, and J. Bever. Frequency-dependent feedback constrains plant community coexistence. Nature Ecology E Evolution 2(9): pp. 1403-1407 (2018). doi:10.1038/s41559-018-0622-3. URL https://doi.org/10.1038\%2Fs41559-018-0622-3

[16] E. Gilad, J. von Hardenberg, A. Provenzale, M. Shachak, and E. Meron. A mathematical model of plants as ecosystem engineers. Journal of Theoretical Biology 244(4): pp. 680-691 (2007). doi:10.1016/j.jtbi.2006. 08.006 .

URL https://doi.org/10.1016\%2Fj.jtbi.2006.08.006

[17] P. Gray and S. Scott. Autocatalytic reactions in the isothermal, continuous stirred tank reactor: isolas and other forms of multistability. Chemical Engineering Science 38(1): pp. 29-43 (1983). doi:10.1016/ 0009-2509(83)80132-8.

URL https://doi.org/10.1016\%2F0009-2509\%2883\%2980132-8

[18] P. Gray and S. Scott. Autocatalytic reactions in the isothermal, continuous stirred tank reactor: oscillations and instabilities in the system A+2B $\rightarrow$ 3B, B $\rightarrow$ C. Chemical Engineering Science 39(6): pp. 1087-1097 (1984). doi:10.1016/0009-2509(84)87017-7. URL https://doi.org/10.1016\%2F0009-2509\%2884\%2987017-7

[19] P. Gray and S. Scott. Sustained oscillations and other exotic patterns of behavior in isothermal reactions. The Journal of Physical Chemistry 89(1): pp. 22-32 (1985). doi:10.1021/j100247a009. URL https://doi.org/10.1021\%2Fj100247a009

[20] J. von Hardenberg, E. Meron, M. Shachak, and Y. Zarmi. Diversity of vegetation patterns and desertification. Physical Review Letters 87(19): p. 198101 (2001). doi:10.1103/physrevlett.87.198101. URL https://doi.org/10.1103\%2Fphysrevlett.87.198101

[21] P. van Heijster and B. Sandstede. Planar radial spots in a three-component FitzHugh-Nagumo system. Journal of Nonlinear Science 21(5): pp. 705-745 (2011). doi:10.1007/s00332-011-9098-x. URL https://doi.org/10.1007/s00332-011-9098-x

[22] G. Hek. Geometric singular perturbation theory in biological practice. Journal of Mathematical Biology 60(3): pp. 347-386 (2010). doi:10.1007/s00285-009-0266-7. URL https://doi.org/10.1007/s00285-009-0266-7

[23] R. Hille Ris Lambers, M. Rietkerk, F. van den Bosch, H. Prins, and H. de Kroon. Vegetation pattern formation in semi-arid grazing systems. Ecology 82(1): pp. 50-61 (2001). doi:10.2307/2680085. URL https://doi.org/10.2307\%2F2680085 
bioRxiv preprint doi: https://doi.org/10.1101/2020.07.29.226522; this version posted June 24, 2021. The copyright holder for this preprint (which was not certified by peer review) is the author/funder. All rights reserved. No reuse allowed without permission.

[24] A. Homburg and B. Sandstede. Homoclinic and heteroclinic bifurcations in vector fields. In Handbook of dynamical systems, Vol. 3, pp. 379-524. North-Holland, Amsterdam (2010). ISBN 978-0-444-53141-4. URL https://wwW.sciencedirect.com/handbook/handbook-of-dynamical-systems/vol/3/ suppl/C

[25] A. Iuorio, N. Popović, and P. Szmolyan. Singular perturbation analysis of a regularized MEMS model. SIAM Journal on Applied Dynamical Systems 18(2): pp. 661-708 (2019). doi:10.1137/18M1197552. URL https://doi.org/10.1137/18M1197552

[26] B. Kealy and D. Wollkind. A nonlinear stability analysis of vegetative Turing pattern formation for an interaction-diffusion plant-surface water model system in an arid flat environment. Bulletin of Mathematical Biology 74(4): pp. 803-833 (2011). doi:10.1007/s11538-011-9688-7.

URL https: //doi.org/10.1007\%2Fs11538-011-9688-7

[27] C. Klausmeier. Regular and irregular patterns in semiarid vegetation. Science 284(5421): pp. 1826-1828 (1999). doi:10.1126/science.284.5421.1826. URL https://doi.org/10.1126/science.284.5421.1826

[28] T. Kolokolnikov, M. J. Ward, and J. Wei. The existence and stability of spike equilibria in the one-dimensional Gray-Scott model: the pulse splitting regime. Physica D: Nonlinear Phenomena 202(3-4): pp. 258-293 (2005). doi:10.1016/j.physd.2005.02.009.

URL https://doi.org/10.1016/j.physd.2005.02.009

[29] T. Kolokolnikov, M. J. Ward, and J. Wei. The existence and stability of spike equilibria in the one-dimensional Gray-Scott model: the low feed-rate regime. Studies in Applied Mathematics 115(1): pp. 21-71 (2008). doi: $10.1111 / \mathrm{j} .1467-9590.2005 .01554$. URL https://doi.org/10.1111/j.1467-9590.2005.01554

[30] C. Kuehn. Multiple Time Scale Dynamics, volume 191 of Applied Mathematical Sciences. Springer (2015). doi:10.1007/978-3-319-12316-5. ISBN 978-3-319-12315-8. URL https://doi.org/10.1007/978-3-319-12316-5

[31] A. Kulmatiski, K. Beard, J. Stevens, and S. Cobbold. Plant-soil feedbacks: a meta-analytical review. Ecology Letters 11(9): pp. 980-992 (2008). doi:10.1111/j.1461-0248.2008.01209.x. URL https://doi.org/10.1111\%2Fj.1461-0248.2008.01209.x

[32] R. Lefever and O. Lejeune. On the origin of tiger bush. Bulletin of Mathematical Biology 59(2): pp. 263-294 (1997). doi:10.1007/bf02462004. URL https://doi .org/10.1007\%2Fbf02462004

[33] Q.-X. Liu, P. Herman, W. Mooij, J. Huisman, M. Scheffer, H. Olff, and J. van de Koppel. Pattern formation at multiple spatial scales drives the resilience of mussel bed ecosystems. Nature Communications 5: p. 5234 (2014). doi:10.1038/ncomms6234. URL https://doi .org/10.1038/ncomms6234

[34] S. Mangan, S. Schnitzer, E. Herre, K. Mack, M. Valencia, E. Sanchez, and J. Bever. Negative plant-soil feedback predicts tree-species relative abundance in a tropical forest. Nature 466(7307): pp. 752-755 (2010). doi:10.1038/nature09273. URL https://doi .org/10.1038\%2Fnature09273

[35] A. Marasco, A. Iuorio, F. Cartenì, G. Bonanomi, D. Tartakovsky, S. Mazzoleni, and F. Giannino. Vegetation pattern formation due to interactions between water availability and toxicity in plant-soil feedback. Bulletin of Mathematical Biology 76: pp. 2866-2883 (2014). doi:10.1007/s11538-014-0036-6. URL https://doi.org/10.1007/s11538-014-0036-6

[36] A. Marciniak-Czochra, S. Härting, G. Karch, and K. Suzuki. Dynamical spike solutions in a nonlocal model of pattern formation. Nonlinearity 31(5): pp. 1757-1781 (2018). doi:10.1088/1361-6544/aaa5dc. URL https://doi .org/10.1088/1361-6544/aaa5dc

[37] A. Marciniak-Czochra, G. Karch, and K. Suzuki. Instability of Turing patterns in reaction-diffusion-ODE systems. Journal of Mathematical Biology 74(3): pp. 583-618 (2017). doi:10.1007/s00285-016-1035-z. URL https://doi .org/10.1007/s00285-016-1035-z 
bioRxiv preprint doi: https://doi.org/10.1101/2020.07.29.226522; this version posted June 24, 2021. The copyright holder for this preprint (which was not certified by peer review) is the author/funder. All rights reserved. No reuse allowed without permission.

[38] S. Mazzoleni, G. Bonanomi, F. Giannino, G. Incerti, S. Dekker, and M. Rietkerk. Modelling the effects of litter decomposition on tree diversity patterns. Ecological Modelling 221(23): pp. 2784-2792 (2010). doi: 10.1016/j.ecolmodel.2010.08.007. URL https://doi.org/10.1016\%2Fj.ecolmodel.2010.08.007

[39] S. Mazzoleni, G. Bonanomi, F. Giannino, M. Rietkerk, S. Dekker, and F. Zucconi. Is plant biodiversity driven by decomposition processes? An emerging new theory on plant diversity. Community Ecology 8(1): pp. 103-109 (2007). doi:10.1556/comec.8.2007.1.12. URL https://doi.org/10.1556\%2Fcomec.8.2007.1.12

[40] S. Mazzoleni, G. Bonanomi, G. Incerti, M. L. Chiusano, P. Termolino, A. Mingo, M. Senatore, F. Giannino, F. Cartenì, M. Rietkerk, and V. Lanzotti. Inhibitory and toxic effects of extracellular self-DNA in litter: a mechanism for negative plant-soil feedbacks? New Phytologist 205(3): pp. 1195-1210 (2015). doi: 10.1111/nph.13121. URL https://doi.org/10.1111/nph.13121

[41] C. Muratov and V. Osipov. Traveling spike autosolitons in the Gray-Scott model. Physica D: Nonlinear Phenomena 155(1-2): pp. 112-131 (2001). doi:10.1016/S0167-2789(01)00259-7. URL https://doi .org/10.1016/S0167-2789(01)00259-7

[42] W. van der Putten, R. Bardgett, J. Bever, T. Bezemer, B. Casper, T. Fukami, P. Kardol, J. Klironomos, A. Kulmatski, J. Schweitzer, K. Suding, T. van de Voorde, and D. Wardle. Plant-soil feedbacks: the past, the present and future challenges. Journal of Ecology 101(2): pp. 265-276 (2013). doi:10.1111/1365-2745. 12054.

URL https://doi .org/10.1111\%2F1365-2745.12054

[43] W. Reynolds, J. Pearson, and S. Ponce-Dawson. Dynamics of self-replicating patterns in reaction diffusion systems. Physical Review Letters 72(17): pp. 2797-2800 (1994). doi:10.1103/physrevlett.72.2797. URL https://doi .org/10.1103\%2Fphysrevlett.72.2797

[44] M. Rietkerk. Self-organized patchiness and catastrophic shifts in ecosystems. Science 305(5692): pp. 19261929 (2004). doi:10.1126/science. 1101867. URL https://doi.org/10.1126\%2Fscience. 1101867

[45] M. Rietkerk, M. Boerlijst, F. van Langevelde, R. Hille Ris Lambers, J. van de Koppel, L. Kumar, H. Prins, and A. de Roos. Self-organization of vegetation in arid ecosystems. The American Naturalist 160(4): p. 524 (2002). doi:10.2307/3079239. URL https://doi .org/10.2307\%2F3079239

[46] M. Rietkerk and J. van de Koppel. Regular pattern formation in real ecosystems. Trends in Ecology $\mathcal{E}$ Evolution 23(3): pp. 169-175 (2008). doi:10.1016/j.tree.2007.10.013. URL https://doi.org/10.1016\%2Fj.tree.2007.10.013

[47] B. de Rijk, A. Doelman, and J. Rademacher. Spectra and stability of spatially periodic pulse patterns: Evans function factorization via Riccati transformation. SIAM Journal on Mathematical Analysis 68(1): pp. 61-121 (2016). doi:10.1137/15M1007264. URL https: //doi .org/10.1137/15M1007264

[48] L. Sewalt and A. Doelman. Spatially periodic multipulse patterns in a generalized Klausmeier-GrayScott model. SIAM Journal on Applied Dynamical Systems 16(2): pp. 1113-1163 (2017). doi:10.1137/ 16M1078756. URL https://doi .org/10.1137/16M1078756

[49] J. Sherratt. Pattern solutions of the Klausmeier model for banded vegetation in semi-arid environments I. Nonlinearity 23(10): pp. 2657-2675 (2010). doi:10.1088/0951-7715/23/10/016. URL https://doi.org/10.1088/0951-7715/23/10/016

[50] J. Sherratt. Pattern solutions of the Klausmeier model for banded vegetation in semi-arid environments II: patterns with the largest possible propagation speeds. Proceedings of the Royal Society A: mathematical, physical and engineering sciences 467(2135): pp. 3272-3294 (2011). doi:doi:10.1098/rspa.2011.0194. URL https://doi .org/doi : 10.1098/rspa.2011.0194 
bioRxiv preprint doi: https://doi.org/10.1101/2020.07.29.226522; this version posted June 24, 2021. The copyright holder for this preprint (which was not certified by peer review) is the author/funder. All rights reserved. No reuse allowed without permission.

[51] J. Sherratt and B. Marchant. Nonsharp travelling wave fronts in the Fisher equation with degenerate nonlinear diffusion. Applied Mathematics Letters 9(5): pp. 33-38 (1996). doi:10.1016/0893-9659(96)00069-9. URL https://doi .org/10.1016/0893-9659(96)00069-9

[52] E. Siero, A. Doelman, M. Eppinga, J. Rademacher, M. Rietkerk, and K. Siteur. Striped pattern selection by advective reaction-diffusion systems: resilience of banded vegetation on slopes. Chaos 35: p. 036411. doi:10.1063/1.4914450. URL http://doi.org/10.1063/1.4914450

[53] M. Simpson, B. Foy, and S. McCue. Travelling waves for a velocity-jump model of cell migration and proliferation. Mathematical Biosciences 24(2): pp. 98-106 (2013). doi:10.1016/j.mbs.2013.04.010. URL https://doi.org/10.1016/j.mbs.2013.04.010

[54] S. van der Stelt, A. Doelman, G. Hek, and J. Rademacher. Rise and fall of periodic patterns for a generalized Klausmeier-Gray-Scott model. Journal of Nonlinear Science 23(1): pp. 39-95 (2013). doi: 10.1007/s00332-012-9139-0.

URL https: //doi .org/10.1007/s00332-012-9139-0

\section{AppendixA. Preparatory asymptotic scaling of system 4.4}

Our goal is to scale system 44.4 in such a way, that we can apply the approach taken in e.g. [13, 14, 48] to construct a travelling pulse solution. For similar asymptotic scaling analyses (with the same goal), see [13, Appendix] and [48, section 2.1].

A priori, every parameter, component, and variable in system (4.4) admits an asymptotic scaling in the small parameter $\varepsilon$. Hence, we scale

$$
\mathcal{A}=\varepsilon^{\alpha} a, \mathcal{B}=\varepsilon^{\beta} b, C=\varepsilon^{\gamma} c, \mathcal{D}=\varepsilon^{\delta} d, \mathcal{H}=\varepsilon^{\eta} h, U=\varepsilon^{\kappa} u, V=\varepsilon^{\lambda} V, S=\varepsilon^{\sigma} s,
$$

and introduce a rescaled coordinate $z=\varepsilon^{\zeta} \tilde{z}$ to obtain

$$
\begin{aligned}
& 0=\varepsilon^{2 \zeta+\kappa} u_{z z}+\varepsilon^{\alpha} a\left(1-\varepsilon^{\kappa} u\right)-\varepsilon^{\kappa+2 \lambda} u v^{2}+\varepsilon^{\gamma+\zeta+\kappa} c u_{z}, \\
& 0=\varepsilon^{2+2 \zeta+\lambda} v_{z z}+\varepsilon^{\kappa+2 \lambda} u v^{2}-\varepsilon^{\beta+\lambda} b v-\varepsilon^{\eta+\lambda+\sigma} h v s+\varepsilon^{\gamma+\zeta+\lambda} c v_{z}, \\
& 0=\varepsilon^{\gamma+\delta+\zeta+\sigma} c d s_{z}-\varepsilon^{\sigma} s+\varepsilon^{\beta+\lambda} b v+\varepsilon^{\eta+\lambda+\sigma} h v s .
\end{aligned}
$$

We now choose $z$ to be the variable on which the (fast) $v$-dynamics take place. Moreover, we assume that the equilibrium $(u, v, s)=\left(\varepsilon^{-\kappa}, 0,0\right)$ is hyperbolic in $v$. This fixes the asymptotic scaling of the coordinate $z$ as

$$
\zeta=\frac{1}{2} \beta-1,
$$

which, dividing the second equation in $\mathrm{A} .2$ by $\varepsilon^{\beta+\lambda}$, yields

$$
\begin{aligned}
& 0=\varepsilon^{\beta-2+\kappa} u_{z z}+\varepsilon^{\alpha} a\left(1-\varepsilon^{\kappa} u\right)-\varepsilon^{\kappa+2 \lambda} u v^{2}+\varepsilon^{\gamma+\frac{1}{2} \beta-1+\kappa} c u_{z}, \\
& 0=v_{z z}+\varepsilon^{\kappa+\lambda-\beta} u v^{2}-b v-\varepsilon^{\eta+\sigma-\beta} h v s+\varepsilon^{\gamma-\frac{1}{2} \beta-1} c v_{z}, \\
& 0=\varepsilon^{\gamma+\delta+\frac{1}{2} \beta-1+\sigma} c d s_{z}-\varepsilon^{\sigma} s+\varepsilon^{\beta+\lambda} b v+\varepsilon^{\eta+\lambda+\sigma} h v s .
\end{aligned}
$$

In the geometric construction as carried out in [13, 14, 48], a pivotal element is the existence of a homoclinic orbit (spike) in $v$ for a fixed value of $u$. In particular, this means that the initial exponential growth close to $v=0$ due to the term $-b v$ in $\mathrm{A} .4 \mathrm{~b}$ must be balanced by a positive, nonlinear term. We fix the asymptotic scaling of $v$ such, that such an homoclinic spike solution has $O(1)$ amplitude in $v$, which implies

$$
\lambda=\beta-\kappa .
$$

With this scaling, dividing the third equation in A.4 by $\varepsilon^{\sigma}$, we obtain

$$
\begin{aligned}
& 0=\varepsilon^{\beta-2+\kappa} u_{z z}+\varepsilon^{\alpha} a\left(1-\varepsilon^{\kappa} u\right)-\varepsilon^{2 \beta-\kappa} u v^{2}+\varepsilon^{\gamma+\frac{1}{2} \beta-1+\kappa} c u_{z}, \\
& 0=v_{z z}+u v^{2}-b v-\varepsilon^{\eta+\sigma-\beta} h v s+\varepsilon^{\gamma-\frac{1}{2} \beta-1} c v_{z}, \\
& 0=\varepsilon^{\gamma+\delta+\frac{1}{2} \beta-1} c d s_{z}-s+\varepsilon^{2 \beta-\kappa-\sigma} b v+\varepsilon^{\eta+\beta-\kappa} h v s .
\end{aligned}
$$


Furthermore, based on the numerical observations from section 3, we conjecture that a homoclinic spike solution in $v$ is, to leading order, symmetric in $z$. This implies that the advection term $c v_{z}$ in the $v$-equation is perturbative, hence

$$
\gamma-\frac{1}{2} \beta-1>0
$$

Next, we consider the behaviour of $u$. For the type of pulse solutions we consider in this paper, there is a clear spatial scale separation between the $u$-component and the $v$-component, see Figure 5 In particular, $u$ is slow in $z$ in comparison to $v$. Rewriting A.6a) as

$$
u_{z z}=\varepsilon^{2+\beta-2 \kappa} u v^{2}-\varepsilon^{2+\alpha-\beta-\kappa} a\left(1-\varepsilon^{\kappa} u\right)-\varepsilon^{1+\gamma-\frac{1}{2} \beta} c u_{z}
$$

this implies that

$$
2+\beta-2 \kappa>0, \quad 2+\alpha-\beta-\kappa>0, \quad 1+\gamma-\frac{1}{2} \beta>0,
$$

where the third inequality already follows from (A.7). Moreover, both from the simulation results presented section 3 and the interpretation of the model 2.4) in light of its dimensional version (2.1), it is clear that the $U$-variable takes values in between the homogeneous dry $(U=0)$ and the homogeneous wet $(U=1)$ states. As $U=\varepsilon^{k} u$, this implies

$$
\kappa \geq 0
$$

For presentation purposes, we henceforth exclude the case $\kappa=0$, to avoid having to treat several scaling subcases independently. However, the analysis in section 4 covers the case $\kappa=0$ as well, as it is nowhere necessary to assume that $\kappa>0$; see also [48, Remarks 1 and 2].

The coupling of $v$ into the $u$-equation plays a central role in the geometric construction outlined in section 4 of this paper, and in the main references [12, 13, 14, 48]. In particular, the question is whether the stable and unstable manifolds of the invariant manifold $C_{0}$ 4.14 intersect transversally, thereby guaranteeing the existence of a (travelling) pulse solution to (2.4). A necessary condition for this intersection to exist, is that the $u v^{2}$-term in A.8 is leading order - that is,

$$
\alpha>2 \beta-\kappa \quad \text { and } \quad \gamma>1+\frac{3}{2} \beta-2 \kappa
$$

Moreover, this term should match, in a specific way, the dynamics of $u$ on $C_{0}$ - that is, the dynamics of $u$ when $v=0$. From A.8, we see that these dynamics are linear, with $u=\varepsilon^{-\kappa}$ being the only equilibrium. This equilibrium is a saddle, with stable resp. unstable eigenvalues

$$
\lambda_{s, u}=\varepsilon^{1+\gamma-\frac{1}{2} \beta}\left(-\frac{c}{2} \pm \sqrt{\varepsilon^{\alpha-2 \gamma} a+\frac{c^{2}}{4}}\right) .
$$

We now assume that there exists an asymptotic scaling such that both eigenvalues (A.12) are nonzero and of the same order, which implies

$$
\alpha-2 \gamma \leq 0
$$

For the same eigenvalues, we consider the associated linear stable and unstable subspaces $E^{s}$ and $E^{u}$ in $\left(u, u_{z}\right)$-phase space. The geometric matching condition now stipulates that the vertical distance between these two subspaces, which is given by

$$
\Delta u_{z}=2\left(1-\varepsilon^{\kappa} u\right) \varepsilon^{1+\gamma-\frac{1}{2} \beta-\kappa} \sqrt{\varepsilon^{\alpha-2 \gamma} a+\frac{c^{2}}{4}},
$$

should be of the same asymptotic order as the leading order term in $\mathrm{A} .8$, that is, $\varepsilon^{2+\beta-2 \kappa} u v^{2}$. We use this condition to fix the scaling of $\mathcal{A}$, yielding

$$
\alpha=2+3 \beta-2 \kappa
$$

This scaling choice further specifies A.6 as

$$
\begin{aligned}
& 0=\varepsilon^{\beta-2+\kappa} u_{z z}+\varepsilon^{2+3 \beta-2 \kappa} a\left(1-\varepsilon^{\kappa} u\right)-\varepsilon^{2 \beta-\kappa} u v^{2}+\varepsilon^{\gamma+\frac{1}{2} \beta-1+\kappa} c u_{z}, \\
& 0=v_{z z}+u v^{2}-b v-\varepsilon^{\eta+\sigma-\beta} h v s+\varepsilon^{\gamma-\frac{1}{2} \beta-1} c v_{z} \\
& 0=\varepsilon^{\gamma+\delta+\frac{1}{2} \beta-1} c d s_{z}-s+\varepsilon^{2 \beta-\kappa-\sigma} b v+\varepsilon^{\eta+\beta-\kappa} h v s .
\end{aligned}
$$

Next, we consider the aim of this paper - that is, to investigate the influence of toxicity on the dynamics of vegetation patterns. The coupling between the toxicity component $s$ and the biomass component $v$ is mediated by 
the term $h v s$. We fix the scaling of the parameter $\mathcal{H}$ such, that the toxicity-coupling term has $O(1)$ influence on the biomass dynamics, which implies

$$
\eta=\beta-\sigma
$$

Applying this scaling choice to system A.16, we obtain

$$
\begin{aligned}
& 0=\varepsilon^{\beta-2+\kappa} u_{z z}+\varepsilon^{2+3 \beta-2 \kappa} a\left(1-\varepsilon^{\kappa} u\right)-\varepsilon^{2 \beta-\kappa} u v^{2}+\varepsilon^{\gamma+\frac{1}{2} \beta-1+\kappa} c u_{z} \\
& 0=v_{z z}+u v^{2}-b v-h v s+\varepsilon^{\gamma-\frac{1}{2} \beta-1} c v_{z} \\
& 0=\varepsilon^{\gamma+\delta+\frac{1}{2} \beta-1} c d s_{z}-s+\varepsilon^{2 \beta-\kappa-\sigma}(b v+h v s) .
\end{aligned}
$$

As a last scaling choice, we consider the question of existence of stationary pulse solutions - that is, when $C=0$ in 4.3 , and as a consequence, $c=0$ in A.18. From the resulting (algebraic) $s$-equation

$$
0=-s+\varepsilon^{2 \beta-\kappa-\sigma}(b v+h v s)
$$

it follows that for a stationary pulse to have a nontrivial $s$-component, the terms in $(A .19)$ all need to have the same asymptotic scaling. When one considers a stationary pulse solution to be a particular travelling pulse solution -namely, one with zero speed- it can be argued that both solution types have to exist within the same asymptotic scaling. Hence, we decide to fix the asymptotic amplitude of $s$ as

$$
\sigma=2 \beta-\kappa
$$

With this scaling, dividing the first equation of $\mathrm{A} .18$ by $\varepsilon^{\beta-2+\kappa}$, we obtain

$$
\begin{aligned}
& 0=u_{z z}+\varepsilon^{4+2 \beta-3 \kappa} a\left(1-\varepsilon^{\kappa} u\right)-\varepsilon^{2+\beta-2 \kappa} u v^{2}+\varepsilon^{1+\gamma-\frac{1}{2} \beta} c u_{z} \\
& 0=v_{z z}+u v^{2}-b v-h v s+\varepsilon^{\gamma-\frac{1}{2} \beta-1} c v_{z} \\
& 0=\varepsilon^{\gamma+\delta+\frac{1}{2} \beta-1} c d s_{z}-s+b v+h v s
\end{aligned}
$$

which is equivalent to the 5-dimensional system (4.7).

The first two equations of system (A.21 for $h=0$ are equivalent to [13, system (A.3)]. Note that in [13], the additional scaling choice $A=\varepsilon^{2} a$ is made - that is, one scaling exponent is specifically chosen to be equal to two. In the context of the scaling presented in this appendix, this would be equivalent to the choice $\beta=\frac{2}{3} \kappa$. Note that this choice specifically precludes the travelling pulse solutions described in Theorem 4.5, see the discussion directly below this Theorem.

In addition, the first two equations of system (A.21) for $h=0$ are equivalent to system [48, system (2.5)]. Note that, in [48], an additional scaling choice is made for the asymptotic scaling of the wave speed. In the context of the scaling presented in this appendix, this would be equivalent to the choice $\gamma=3+\frac{3}{2} \beta-\kappa$, which implies $\varepsilon_{3}=\varepsilon_{1}$, see 4.9, 4.36. This choice for the asymptotic scaling of the wave speed is different from the result $\gamma=1+\frac{3}{2} \beta-\kappa$ presented in Theorem 4.5, the two choices coincide only if $\kappa=2$, which is a subcase of Theorem 4.5 . 